\title{
Two-dimensional turbulence properties of the ECMWF reanalyses
}

\author{
By DAVID M. STRAUS ${ }^{1 *}$ and PETER DITLEVSEN ${ }^{2},{ }^{1}$ Center for Ocean-Land-Atmosphere Studies, \\ 4041 Power Mill Road, Suite 302, Calverton, MD 20705, USA; ${ }^{2}$ The Niels Bohr Institute, Geophysical \\ Department, Juliane Maries Vej 30, DK-2100 Copenhagen Ø, Denmark
}

(Manuscript received 14 September 1998; in final form 17 June 1999)

\begin{abstract}
A spherical harmonic analysis is made of the stationary and transient rotational motions during 14 winter and 15 summer seasons from the reanalyses of the European Centre for MediumRange Weather Forecasts (ECMWF) for the northern hemisphere. Vertically-integrated kinetic energy, enstrophy, rotational non-linear interactions and the baroclinic source term are diagnosed as a function of total wavenumber $n$. The contributions to the non-linear transfers from triads involving wavenumber and frequency bands of meterological relevance are mapped. The inter-seasonal and intra-seasonal variability are computed. The non-linear energy and enstrophy tendencies and fluxes are examined and compared to existing geophysical turbulence theories. The transient divergent kinetic energy is less than the rotational energy. The spectral energy slope seen in the range $n \sim 10-40$ is roughly $-2.5 \sim-2.6$. Based on the variability of the slope on seasonal and 10-day time scales, this slope is significantly different than -3 . There is no indication of the $-5 / 3$ mesoscale energy regime seen in observations. A broad enstrophy dissipation regimes is seen for $n>40$. Non-linear terms transfer transient energy from a band centered at $n \sim 15$ to one at $n \sim 7$, with the latter predominantly associated with non-zonal (zonal wavenumber $m \neq 0$ ) flow. Non-linear terms transfer mean energy from $n=7$ to the mean zonal flow $m=0, n=3$ and $n=5$. The non-linear transfer of transient energy is quite variable, with about $16 \%$ of 10 -day periods yielding a tendency twice the mean, and $16 \%$ showing no upscale tendency whatever. This variability is greatly reduced when interactions involving only synoptic scales $(n \sim 10-40)$ are retained. The latter set of interactions are associated mostly with triads involving both high and low frequencies, with associated periods in the 1-9 day and 11-90 range, respectively. Non-local planetary wave advective interactions play an important rôle in the downscale transfer of enstrophy. More local interactions involving synoptic scales dominate the non-linear energy transfers. The main seasonal effects are a weakening in summer of the total energy and shifting to higher wave number of the peak, and a distinct shift to smaller scales in the transition between the large-scale and synoptic-scale regimes in the energy budget. The predominant time scale for non-linear maintenance of the planetary waves (about 9 days) is roughly the same as that of the baroclinic support of larger synoptic scale waves. The time scale of baroclinic conversion which maintains the smaller synoptic waves $(n \sim 20-40)$ is shorter (about 4 days).
\end{abstract}

\section{Introduction}

The strong tendency of non-linear 2-dimensional dynamics to transfer energy to large scales

\footnotetext{
* Corresponding author. e-mail: straus@cola.iges.org
}

(Fjørtoft, 1953) is responsible for widespread interest in the application of 2-dimensional turbulence to the general circulation of the atmosphere. Since the upscale transfer of energy from synoptic to planetary scales is a potentially important process in understanding large-scale atmospheric variability, early studies focused on the diagnosis of this 
transfer from atmospheric data (Saltzman and Fleisher, 1960; Steinberg and Wiin-Nielsen, 1971; Chen and Wiin-Nielsen, 1978; Lambert, 1981). With the recognition that quasi-geostrophic dynamics share a fundamental property with 2-dimensional incompressible dynamics (they both conserve forms of energy and vorticity), it became clear that quasi-geostrophic turbulence was highly relevant to geophysical fluid dynamics in general and the atmosphere in particular (Charney, 1971; Rhines, 1979).

At the same time, the critical importance of the energy spectrum in understanding atmospheric predictability (first pointed out by Lorenz, 1969) provided another link between atmospheric dynamics and those of turbulence. Lorenz pointed out that an energy spectrum proportional to $k^{-3}$ (with $k$ being horizontal wavenumber) formed a significant regime boundary with regard to predictability. A spectrum associated with a slope of -3 or steeper was associated with a flow whose predictability at any fixed range could be increased indefinitely by reducing the initial error. However, a spectrum whose slope was less steep than -3 implied a flow which was fundamentally less predictable, for the error at any fixed range could no longer be reduced below a (finite) value no matter how small the initial error was made. Not only does the simplest scaling theory of 2-dimensional (and quasi-geostrophic) turbulence predict a -3 spectrum (see the review of Orszag (1977)), but a number of early observational studies suggested that this spectrum holds approximately for the synoptic to large scales in the atmosphere (Horn and Bryson, 1963; Wiin-Nielsen, 1967, 1972; Julian et al, 1970; Kao and Wendell, 1970). Leith (1971) and Leith and Kraichnan (1972) put these ideas on a firm theoretical base with the use of turbulence closure models in the context of atmosphericlike forcing.

These early successes stimulated a very large number of theoretical papers exploring the relationships between 2-dimensional and quasi-geostrophic turbulence and atmospheric transient flow (Rhines, 1975; Salmon, 1980; Basdevant et al, 1981; Hoyer and Sadourny, 1982; Holloway, 1982; Vallis, 1983). Of course interactions with the time mean flow are also known to be of great importance in determining the structure of the transients (Hoskins, 1983). Even in the barotropic case, the effects on the transients of a mean zonal jet distorts the nature of the non-linear turbulent interactions (Shepherd, 1987b). In the more general baroclinic case, the interplay between the development of transients in a mean vertical shear (baroclinic instability) and quasi-geostrophic turbulence was studied in fairly idealized contexts by Salmon (1980), Haidvogel and Held (1980), Hoyer and Sadourny (1982), Vallis (1983) and Hua and Haidvogel (1986). Utilizing the vertical mode framework first introduced by Charney (1971), Salmon (1981), Hoyer and Sadourny (1982), and Hua and Haidvogel (1986) arrived at the same qualitative picture. The transfer of total (kinetic plus available potential) energy to larger scales is achieved entirely by the first vertical (barotropic) mode, with the transfer of enstrophy to smaller scales achieved both by the barotropic and higher (baroclinic) modes. At the Rossby radius of deformation, baroclinic processes transfer energy primarily from the first baroclinic mode to the barotropic mode. Thus in the context of barotropic dynamics, baroclinic instablity provides the input for the turbulent energy and enstrophy cascades.

Recent observational studies of aircraft data (Nastrom and Gage, 1985, hereafter NG; Högström et al., 1999) have re-energized the discussion by finding not only a -3 spectral range for kinetic energy and temperature at synoptic scales $(\sim 1000-4000 \mathrm{~km})$ but a $-5 / 3$ spectral range at mesoscales $(\sim 100-800 \mathrm{~km})$. Kraichnan $(1967,1971)$ gave strong arguments that isotropic homogenous 2-dimensional turbulence forced at "intermediate" wavenumbers can support an energy cascade towards larger scales ("upscale") associated with a $-5 / 3$ spectrum and no enstrophy transfer, and at the same time an enstrophy cascade towards smaller scales ("downscale"), associated with a -3 spectrum and no energy transfer. But in this scenario the $-5 / 3$ spectrum appears at larger scales than the -3 spectrum, which is inconsistent with the observations. Isotropic and homogeneous 3-dimensional turbulence predicts a $-5 / 3$ spectrum, but is expected to come into play only at much smaller scales than the mesoscales. A number of investigations have attempted to theoretically explain the observations by suggesting the presence of two sets of 2-dimensional turbulence inertial ranges (Lilly, 1983, 1989; Gage and Nastrom, 1986; Gifford, 1988; Bartello, 1995; Vallis, 1997). The large scale kinetic energy source associated with 
baroclinic conversion from available potential energy gives rise to the downscale -3 enstrophy cascading range, while a source associated with latent heat release at scales $\sim 100 \mathrm{~km}$ gives rise to a combination of gravity wave propagation and 2-dimensional upscale energy propagation, the dimensionality of the latter suggested by the highly stratified nature of convective outflows. While there is some agreement that the spectrum of vertical velocity is characteristic of gravity wave propagation, while the horizontal wind and temperature spectra are more characteristic of turbulence (see Högström et al. (1999) for a discussion), the interplay of waves and turbulence in this context is not well understood.

The extensive use of atmospheric analyses based on 4-dimensional data assimilation to study the energy spectra and the non-linear energy and enstrophy fluxes in terms of total wavenumber $n$ was enhanced by the work of Boer (1983), Boer and Shepherd (1983), Shepherd (1987a) and Boer (1994). Building on earlier work of Wiin-Nielsen (1967, 1972) and Baer (1972), they developed a complete barotropic framework for the discussion of the rotational energy and enstrophy transfers of the mean flow, the transient flow, and their interaction, and used this framework to analyze global data from January and July of 1979. In the barotropic framework the baroclinic energy conversion resulting from baroclinic development is treated as a source term for kinetic energy, although it must be recognized that this source is in fact determined by the flow itself.

Boer and Shepherd (1983) show a spectral slope of -3 for the transient kinetic energy near the jet stream level $(200-300 \mathrm{hPa})$, but a smaller slopes at other levels. They also present evidence that the transient eddy statistics for $n>10$ are consistent with isotropy and homogeneity to a surprising degree, in apparent contradiction to the work of Hoskins et. al. (1983) distinguishing between the shape of low- and high-frequency transient eddies. Shepherd (1987a) stressed that the turbulence paradigm is most applicable to the transient interactions, and that the interactions between transient and mean flow are best understood by studying each zonal wavenumber $m$ separately. A flux of transient rotational kinetic energy upscale from $n \sim 15$ to lower values, and a downscale enstrophy transfer from $n \sim 15$ to larger values are found. These transfers are consistent with those expected for 2-dimensional turbulence arguments in conjunction with a baroclinic energy source near $n \sim 15$. The fluxes are by no means constant over any range of wavenumbers, indicating the absence of a true inertial range. A caveat to this conclusion is that only global scales $n<40$ were analyzed.

Higher resolution data sets were examined by Trenberth and Solomon (1993), who computed the kinetic energy and temperature spectra for several Januaries from analyses at spectral resolution of T106. In agreement with the work of Boer and Shepherd, they found slopes approaching -3 only in the upper troposphere, with more shallow spectra below and in the stratosphere. These slopes held over a wavenumber range of $n \sim 20-60$. At smaller scales dissipation takes over and the spectra become much steeper. Koshyk and Boer (1995) explored the resolution dependence of the energy and enstrophy transfers for very high global resolution analyses (up to $n=213$ ). Increasing resolution leads to a larger upscale energy transfer and a larger downscale enstrophy transfer, although again there is little evidence for true energy or enstrophy cascades.

The lack of an upscale energy cascading inertial range is hardly surprising. On the one hand the theoretical arguments of Kraichnan (1967, 1971) require the presence of an asymptotically infinite range of scales larger than the energy injection scale; even a large but finite range gives rise to competing energy (and enstrophy) transfers in the "wrong" direction (Terry and Newman, 1993). In the real atmosphere, on the other hand, the injection scale corresponds to $n \sim 15$, so there is a very small available range for up-scale transfer, a configuration far from that assumed by Kraichnan. Yet the upscale energy transfer that does exist in the atmosphere is of intrinsic interest, for it provides a source of large scale (and potentially low frequency) variability. (Conditions in the ocean are quite different, where the far smaller ratio of the radius of deformation to the largest scales makes the cascade theory more applicable, as for example in Hoyer and Sadourny, 1982).

The main purpose of this paper is to study the properties of this upscale energy transfer in a realistic representation of the real atmosphere. While turbulence theory would suggest that the interactions that give rise to this transfer are nonlocal in wavenumber space, it is of interest to understand the spatial and temporal scales that 
participate in these interactions, for this may suggest what meteorological phenomena are involved. Another question of interest relates to the robustness of the upscale energy transport on both intraseasonal and interannual time scales. The climatological differences between winter and summer energy transports are also relevant in understanding this source of large scale atmospheric variability. In the theory of (nearly inviscid) 2-dimensional turbulence, the downscale enstrophy cascade is based to a large extent on interactions which are non-local in wavenumber space, i.e., interactions involving groups of waves with very different $n$. It is of interest to examine the local and non-local interactions between various meterological scales.

The representation of the real atmosphere which we use to address these questions is the set of reanalyses of the European Centre for MediumRange Weather Forecasts (ECMWF, Gibson et al., 1997). This set of reanalyses was carried out consistently for 15 years, and nominally retains all scales $n \leqslant 106$. Given this degree of resolution, we might expect some indication of a change from an approximately -3 (large-scale) spectrum to the mesoscale $-5 / 3$ spectrum. However, it is not clear that scales smaller than $1000 \mathrm{~km}$ can really be resolved (Lander and Hoskins, 1997). Further, the dissipative nature of the semi-Lagrangian numerical scheme used in the forecast model, and the possible involvement of mesoscale gravity waves in the dynamics of the $-5 / 3$ regime (see earlier cited references) makes it unlikely that this spectrum will be seen in the reanalyses. The work of Trenberth and Solomon (1993) using the ECMWF operational analyses certainly gives no hint of a shallower spectrum at small scales. It is nevertheless of interest to examine the spectrum for all resolved wavenumbers.

The diagnostic method we apply to the reanalyses is based on 2-dimensional turbulence theory (as in Boer and Shepherd, 1983), in which baroclinic development enters the barotropic vorticity equation via the kinetic energy source term. However, since we vertically integrate all terms in the 2-dimensional energy $(E)$ and enstrophy $(Z)$ budgets, correlations between baroclinic modes which contribute to the vertically averaged $E$ and $Z$ equations are taken into account. Thus our approach is equivalent to a complete barotropic mode analysis for quasi-geostrophic turbulence (as for example in Hua and Haidvogel, 1986).

In this study, we utilize 14 winter and 15 summer seasons (each of 90 days), so that the total size of the data base is $45 \times$ that of previous studies which have examined a single winter and summer month (30 days). We concentrate on the northern hemisphere so that the question of seasonality can be meaningfully addressed.

In terms of spatial scales, we consider several groups of wavenumbers which encompass broad meterological categories: (1) The planetary wave group encompassing the largest scales (total wavenumber $n=1-10$ ) which are forced by topography, land-sea heat contrast, and sea surface temperature anomalies, and whose variability is related to global Rossby waves, regime-like transitions in mid-latitude flow, and large scale tropical waves. (2) The synoptic wave group, which includes scales of total wavenumbers $n=10-40$. The larger scales in the group $(n=10-20)$ describe in broad terms important stationary Rossby wave propagation and baroclinic development, while the smaller scales in this group $(n=20-40)$ give the signature of fronts and more detailed structures in cyclones. (3) The true small-scale group $(n=$ 40-106) describes more local circulations which are likely to be most sensitive to model parameterizations of dissipation and heating. The composition of these groups is motivated by the behavior of the energy spectrum, to be discussed.

The temporal scales for the transient flow are described by two components: a high-frequency component which includes fluctuations with periods of 1 to 9 days, and a low-frequency component including fluctuations of about 11 to 90 days. The high-frequency component is generally associated with mobile atmospheric disturbances which tend to be concentrated in the storm track regions, while the low-frequency fluctuations describe the environment in which the disturbances grow, propagate, and decay. The non-linear interactions between different spatial scales (see above) will be further studied in terms of selfinteractions of the high-frequency components, self-interactions of the low-frequency components, and interactions between the two frequency groups.

The outline of this papers is as follows: Section 2 briefly describes the reanalysis data set and the data processing. A full account of the equations 
of the turbulence (spherical harmonic) framework as applied to the transient and stationary flow is given in Section 3. In Section 4 we give the results for the transient and mean flow kinetic energy spectra for rotational and divergent flow, and for winter and summer, and in Section 5 discuss the upscale energy transfer and the downscale enstrophy transfer. A summary (Section 6) and discussion (Section 7) follow.

\section{Data processing}

The ECMWF Re-Analysis Project (ERA) produced a validated 15-year data set of assimilated data for the period of 1979 through 1993 (Gibson, et al., 1997). The data assimilation system used the Integrated Forecast System version of the ECMWF forecast model with 3-dimensional semiLagrangian advection, a spectral truncation of triangular 106 (T106), and 31 vertical hybrid levels. Prognostic equations for cloud water, ice content and cloud cover were included. Data were assimilated with an intermittent statistical optimal interpolation technique using a $6 \mathrm{~h}$ cycling time. One-dimensional variational physical retrieval of the TOVS* cloud cleared radiances was used below $100 \mathrm{hPa}$. For further details consult Gibson et al. (1997).

We utilized twice-daily time series of the spectral coefficients of vorticity and divergence at the following 10 analysis levels: 850, 700, 600, 500, $400,300,250,200,150$ and $100 \mathrm{hPa}$. The winter (summer) season was defined as the 90 -day period commencing on 00 UTC 21 December (June). For each analysis time the northern hemisphere data were reflected about the equator to produce "global" data. Since the $u(v)$ wind has even (odd) parity with respect to reflection about the equator, the following procedure was used to insure a smooth wind field in the immediate vicinity of the equator: Between $5^{\circ} \mathrm{N}$ and the equator at each longitude the zonal wind $u$ was replaced by a polynomial in latitude which preserved the first derivative of the field at $5^{\circ} \mathrm{N}$ but had vanishing derivative at the equator. For the meridional wind

* The TIROS Operational Vertical Sounder (TOVS) measures multi-spectral radiances, which are related to the temperature and humidity structure in the atmosphere. $v$ the polynomial preserved the first derivative at $5^{\circ} \mathrm{N}$ but vanished at the equator.

The groups of spatial scales are based on total wavenumber $n$, and are given in detail in the next section. In terms of temporal scales, the annual cycle was calculated from a parabolic fit for each 90-day season separately (as in Straus, 1983). For each season, the turbulence diagnostics to be presented below were calculated for the stationary (time mean flow); for the transient flow we used four separate definitions.

(i) Total transients - deviations from current seasonal mean.

(ii) Annual cycle transients - deviations from current annual cycle.

(iii) Low-frequency transients - low-frequency deviations from current annual cycle.

(iv) High-frequency transients - high-frequency deviations from current annual cycle.

The low- and high-frequency transients were calculated from a discrete Fourier transform in time, with the former comprising fluctuations of periods 90 down to 11.25 days, and the latter periods of 9 days down to 1 day. The interactions between low- and high-frequency transients (to be discussed below) were obtained from (ii)-(iv). All the terms kept in the turbulence analysis were computed separately for every 10-day period. The inter-seasonal variability of any quantity is then given by $\sigma_{1}^{2}$, the variance of seasonal means about climatology, while the intra-seasonal variability is given by $\sigma_{2}^{2}$, the variance of 10-day means about the current seasonal mean.

\section{Spherical harmonic turbulence analysis}

This analysis recapitulates the development of Boer and Shepherd (1983), Shepherd (1987a) and Boer (1994). We start from the mean and transient vorticity equations:

$\frac{\partial \bar{\zeta}}{\partial t}=\bar{\zeta}^{(\mathrm{L})}+\bar{\zeta}^{(\mathrm{N})}+\bar{S}$,
$\frac{\partial \zeta^{\prime}}{\partial t}=\zeta^{\prime(\mathrm{L})}+\zeta^{\prime(\mathrm{N})}+S^{\prime}$,

where $\zeta$ is the vorticity, an overbar denotes the seasonal mean, and the prime denotes a transient departure from that seasonal mean, corresponding 
to the total transient designation of the previous section. The linear terms $\bar{\zeta}^{(\mathrm{L})}$ and $\zeta^{(\mathrm{L})}$ are:

$\bar{\zeta}^{(\mathrm{L})}=-\nabla \cdot(\bar{v} f)$,

$\zeta^{\prime(\mathrm{L})}=-\nabla \cdot\left(\overline{\boldsymbol{v}} \zeta^{\prime}\right)-\nabla \cdot\left(\boldsymbol{v}^{\prime} \bar{\zeta}\right)-\nabla \cdot\left(\boldsymbol{v}^{\prime} f\right)$,

with $f=2 \Omega \sin (\phi)$, where $\phi$ is latitude and $\Omega$ the rotation rate of the earth. In general the velocity field $\vec{v}$ is written in the usual way as the sum of the rotational and divergent parts as:

$\boldsymbol{v}=\hat{\boldsymbol{k}} \times \nabla \psi+\nabla \chi$.

Here $\psi$ is the streamfunction, $\chi$ the velocity potential, and $\hat{\boldsymbol{k}}$ the unit vector in the vertical direction. We include only the rotational part of the velocity in the mean and transient vorticity equation. (To consistently include the divergent part, we would also have to include terms involving the vertical velocity, according to standard quasi-geostrophic scaling.)

The non-linear terms $\bar{\zeta}^{(\mathrm{N})}$ and $\zeta^{\prime(\mathrm{N})}$ are:

$\bar{\zeta}^{(\mathrm{N})}=-\nabla \cdot(\overline{\boldsymbol{v}} \bar{\zeta})-\nabla \cdot\left(\overline{\boldsymbol{v}^{\prime} \zeta^{\prime}}\right)=\bar{\zeta}^{\left(\mathrm{N}_{1}\right)}+\bar{\zeta}^{\left(\mathrm{N}_{2}\right)}$,

$\zeta^{\prime(\mathrm{N})}=-\nabla \cdot\left(\boldsymbol{v}^{\prime} \zeta^{\prime}\right)+\overline{\nabla \cdot\left(\boldsymbol{v}^{\prime} \zeta^{\prime}\right)}$.

In eq. (6) we have explicitly labeled the non-linear contribution of the mean flow $\left(\mathrm{N}_{1}\right)$ and that due to the transient flow effect on the mean flow $\left(\mathrm{N}_{2}\right)$. In eq. (7) the mean correction due to the transients (second term) plays no rôle in the kinetic energy and enstrophy budgets.

The residual (or source) terms $\bar{S}$ and $S^{\prime}$ include all effects of the divergent component of the flow, including vertical advection and the "tilting" term, frictional effects, and since we are applying the vorticity equation to analyses, a source term due to insertion of the observational data.

We expand the scalar fields $(\psi, \chi, \zeta$, and the divergence $D$ ) in terms of spherical harmonics, as for example:

$\psi(\lambda, \mu)=\sum_{n=0}^{n=N} \sum_{m=-n}^{m=n} P_{n, m}(\mu) \mathrm{e}^{i m \lambda} \psi_{n, m}$,

where $\lambda$ is longitude, $\phi$ latitude, $\mu=\sin (\phi), n$ the total wave number, and $m$ the zonal wave number. $P_{n, m}$ is the associated Legendre polynomial, and $\psi_{n, m}$ is a complex coefficient. See Boer and Shepherd (1983) for more details. Since the pairs $(\psi, \zeta)$ and $(\chi, D)$ are related by:

$\zeta=\nabla^{2} \psi$,

$D=\nabla^{2} \chi$, we also have:

$\zeta_{n, m}=-\frac{n(n+1)}{a^{2}} \psi_{n, m}$,
$D_{n, m}=-\frac{n(n+1)}{a^{2}} \chi_{n, m}$,

where $a$ is the radius of the earth.

The globally averaged kinetic energy is given by:

$$
\begin{aligned}
E & =-\frac{1}{2}\langle\overline{\psi \zeta}\rangle=-\frac{1}{2}\langle\bar{\psi} \bar{\zeta}\rangle-\frac{1}{2}\left\langle\overline{\psi^{\prime} \zeta^{\prime}}\right\rangle \\
& =-\frac{1}{4} \sum_{n=0}^{n=N} \sum_{m=-n}^{m=n}\left(\bar{\psi}_{n, m} \bar{\zeta}_{n, m}^{*}+\overline{\psi_{n, m}^{\prime}+\zeta_{n, m}^{\prime *}}\right) \\
& =+\frac{1}{4} \sum_{n=0}^{n=N} \frac{n(n+1)}{a^{2}} \sum_{m=-n}^{m=n}\left(\bar{\psi}_{n, m} \bar{\psi}_{n, m}^{*}+\overline{\psi_{n, m}^{\prime} \psi_{n, m}^{\prime *}}\right) \\
& =\sum_{n=0}^{n=N}\left(\bar{E}_{n}+E_{n}^{\prime}\right)
\end{aligned}
$$

$\bar{E}_{n}$ and $E_{n}^{\prime}$ can be written as:

$\bar{E}_{n}=\frac{1}{2} \frac{n(n+1)}{a^{2}}\left(\frac{1}{2} \bar{\psi}_{n, 0}^{2}+\sum_{m=1}^{m=n} \bar{\psi}_{n, m} \bar{\psi}_{n, m}^{*}\right)$,

$E_{n}^{\prime}=\frac{1}{2} \frac{n(n+1)}{a^{2}}\left(\frac{1}{2} \overline{\psi_{n, 0}^{\prime 2}}+\sum_{m=1}^{m=n} \overline{\psi_{n, m}^{\prime} \psi_{n, m}^{\prime *}}\right)$.

In these equations, the angular brackets denote a global average, and the asterisk denotes complex conjugate. The extra factor of $\frac{1}{2}$ in eq. (14) arises from the choice of normalization of the Legendre polynomials. An entirely analagous development holds for the divergent kinetic energy, with $\chi$ replacing $\psi$ and $D$ replacing $\zeta$.

The globally averaged enstrophy $Z$ can be written as:

$$
\begin{aligned}
Z & =\frac{1}{2}\langle\overline{\zeta \zeta}\rangle=\frac{1}{2}\langle\bar{\zeta} \bar{\zeta}\rangle+\frac{1}{2}\left\langle\overline{\zeta^{\prime} \zeta^{\prime}}\right\rangle \\
& =\frac{1}{4} \sum_{n=0}^{n=N} \sum_{m=-n}^{m=n}\left(\bar{\zeta}_{n, m} \bar{\zeta}_{n, m}^{*}+\overline{\zeta_{n, m}^{\prime} \zeta_{n, m}^{\prime *}}\right) \\
& =\frac{1}{4} \sum_{n=0}^{n=N}\left(\frac{n(n+1)}{a^{2}}\right)^{2} \sum_{m=-n}^{m=n}\left(\bar{\psi}_{n, m} \bar{\psi}_{n, m}^{*}+\overline{\psi_{n, m}^{\prime} \psi_{n, m}^{\prime *}}\right) \\
& =\sum_{n=0}^{n=N} \frac{n(n+1)}{a^{2}}\left(\bar{E}_{n}+E_{n}^{\prime}\right) \\
& =\sum_{n=0}^{n=N}\left(\bar{Z}_{n}+Z_{n}^{\prime}\right)
\end{aligned}
$$


so that the enstrophy components are simply the corresponding energy components multiplied by the factor $n(n+1) / a^{2}$. Now eqs. (1) and (2) can be transformed into spectral space to yield:

$$
\begin{aligned}
\frac{\mathrm{d} \bar{\zeta}_{n, m}}{\mathrm{~d} t} & =\bar{\zeta}_{n, m}^{(\mathrm{L})}+\bar{\zeta}_{n, m}^{(\mathrm{N})}+\bar{S}_{n, m} \\
& =\bar{\zeta}_{n, m}^{(\mathrm{L})}+\bar{\zeta}_{n, m}^{\left(\mathrm{N}_{1}\right)}+\zeta_{n, m}^{\left(\mathrm{N}_{2}\right)}-\bar{S}_{n, m}, \\
\frac{\mathrm{d} \zeta_{n, m}^{\prime}}{\mathrm{d} t} & =\zeta_{n, m}^{\prime(\mathrm{L})}+\zeta_{n, m}^{\prime(\mathrm{N})}+S_{n, m}^{\prime}
\end{aligned}
$$

with equivalent equations for $\psi_{n, m}$. Here we have used the notation of eq. (6).

From the time derivative of eqs. (17) and (18), utilizing eqs. (24) and (25) we have:

$$
\begin{aligned}
\frac{\mathrm{d} \bar{E}_{n}}{\mathrm{~d} t} & =X_{n}^{\mathrm{L}}+X_{n}^{N}+X_{n}^{\mathrm{S}} \\
& =X_{n}^{\mathrm{L}}+\left(X_{n}^{\mathrm{N}_{1}}+X_{n}^{\mathrm{N}_{2}}\right)+X_{n}^{\mathrm{S}}, \\
\frac{\mathrm{d} E_{n}^{\prime}}{\mathrm{d} t} & =T_{n}^{\mathrm{L}}+T_{n}^{\mathrm{N}}+T_{n}^{\mathrm{S}} .
\end{aligned}
$$

Here $X^{\mathrm{L}}$ is the mean flow energy tendency due to the Coriolis term, $X^{\mathbf{N}_{1}}$ is due to non-linear interactions of the mean flow and $X^{\mathrm{N}_{2}}$ due to the transient feedback on the mean flow. $T^{\mathrm{L}}$ is the rate of transient energy change due to interactions with the mean flow and $T^{\mathrm{N}}$ the rate of energy change due to transient non-linear interactions. The terms labeled with $\mathrm{S}$ are the source terms including vertical flux terms, baroclinic conversion, dissipation and analysis increments.

For use in the later development, it is helpful to note that since the term $\zeta_{n, m}^{\prime(\mathrm{N})}$ in eq. (25) is itself non-linear in the transients, the term $T^{\mathrm{N}}$ is a triple moment composed of triad interactions. Thus if the transients are partitioned into low- and highfrequency components (see the previous section), the time rate of change of energy in each component due to the non-linear interactions will involve not only that component but interactions with the other component.

From eqs. (23) and the equivalent to eqs. (17) and eqs. (18) for $\bar{Z}_{n}$ and $Z_{n}^{\prime}$ we can also write

$$
\begin{aligned}
\frac{\mathrm{d} \bar{Z}_{n}}{\mathrm{~d} t} & =Y_{n}^{\mathrm{L}}+Y_{n}^{\mathrm{N}}+Y_{n}^{\mathrm{S}} \\
& =Y_{n}^{\mathrm{L}}+\left(Y_{n}^{\mathrm{N}_{1}}+Y_{n}^{\mathrm{N}_{2}}\right)+Y_{n}^{\mathrm{S}}, \\
\frac{\mathrm{d} Z_{n}^{\prime}}{\mathrm{d} t} & =Q_{n}^{\mathrm{L}}+Q_{n}^{\mathrm{N}}+Q_{n}^{\mathrm{S}},
\end{aligned}
$$

where each term in eqs. (29), (30) and (31) is related to the corresponding terms in eqs. (26), (27) and (28) by the factor $n(n+1) / a^{2}$.

Kinetic energy is conserved not only by the globally averaged adiabatic tendency terms, but also by the globally averaged non-linear terms; the terms involving the Coriolis parameter vanish when summed over $n$. Thus the sum over all wavenumbers $n$ of each of the following terms vanishes: $X_{n}^{\mathrm{N}_{1}}, T_{n}^{\mathrm{N}}$, and $T_{n}^{\mathrm{L}}+X_{n}^{\mathrm{N}_{2}}$, where the third term includes all non-linear (mean/transient) interaction terms. Enstrophy conservation similarly leads to the vanishing of the sum of: $Y_{n}^{\mathrm{N}_{1}}, Q_{n}^{\mathrm{N}}$, and $Q_{n}^{\mathrm{L}}+Y_{n}^{\mathrm{N}_{2}}$.

For each conserved quantity we can define a corresponding flux, which gives the rate at which that quantity is transferred from wavenumbers less than $n$ to wavenumbers greater than $n$. Corresponding to $T^{\mathrm{N}}$ and $Q^{\mathrm{N}}$ we have the transient energy and enstrophy fluxes, $F_{n}$ and $H_{n}$ respectively):

$$
\begin{aligned}
& F_{n+1}=-\frac{1}{a} \sum_{n^{\prime}=1}^{n^{\prime}=n} T_{n^{\prime}}^{\mathrm{N}} \\
& H_{n+1}=-\frac{1}{a} \sum_{n^{\prime}=1}^{n^{\prime}=n} Q_{n^{\prime}}^{\mathrm{N}}
\end{aligned}
$$

so that

$T_{n}^{\mathrm{N}}=-a\left(F_{n}-F_{n-1}\right)$,

$Q_{n}^{\mathrm{N}}=-a\left(H_{n}-H_{n-1}\right)$.

The triad interactions implicit in $T_{n}^{\mathrm{N}}$ and $Q_{n}^{\mathrm{N}}$ are mapped in terms of broad categories by the following scheme. First the transient vorticity is divided into wave groups. In position space this division is written as:

$\zeta^{\prime}=\zeta_{\mathrm{P}}+\zeta_{\mathrm{S}}+\zeta_{\mathrm{s}}$

where the subscripts are:

- P planetary waves (global wave number $0 \leqslant n \leqslant 10$ )

- $\mathrm{S}$ synoptic waves (global wave number $11 \leqslant n \leqslant 40$ )

- s small-scale waves (global wave number $41 \leqslant n \leqslant 106$ )

Based on this division, the term $\zeta_{n, m}^{\prime(\mathrm{N})}$ in eq. (25) can then be written as the sum of 6 terms:

$\zeta^{\prime(\mathrm{N})}=\zeta_{\mathrm{P} / \mathrm{P}}^{(\mathrm{N})}+\zeta_{\mathrm{S} / \mathrm{S}}^{(\mathrm{N})}+\zeta_{\mathrm{s} / \mathrm{s}}^{(\mathrm{N})}+\zeta_{\mathrm{P} / \mathrm{S}}^{(\mathrm{N})}+\zeta_{\mathrm{P} / \mathrm{s}}^{(\mathrm{N})}+\zeta_{\mathrm{S} / \mathrm{s}}^{(\mathrm{N})}$

where we have suppressed the indices $n$ and $m$, 
and this breakdown details the interactions within each group and between groups. Substituting this expansion into eq. (25) leads to a similar expansion for $T_{n}^{\mathrm{N}}$ in eq. (28):

$$
\begin{aligned}
T_{n}^{\mathrm{N}}= & T_{n(\mathrm{P} / \mathrm{P})}^{\mathrm{N}}+\left(T_{n(\mathrm{P} / \mathrm{S})}^{\mathrm{N}}+T_{n(\mathrm{P} / \mathrm{s})}^{\mathrm{N}}\right) \\
& +T_{n(\mathrm{~S} / \mathrm{S})}^{\mathrm{N}}+T_{n(\mathrm{~S} / \mathrm{s})}^{\mathrm{N}}+T_{n(\mathrm{~s} / \mathrm{s})}^{\mathrm{N}} \\
= & T_{n(\mathrm{P} / \mathrm{P})}^{\mathrm{N}}+T_{n}^{\mathrm{N}_{\mathrm{A}}}+T_{n}^{\mathrm{N}_{\mathrm{B}}}+T_{n}^{\mathrm{N}_{\mathrm{C}}}+T_{n(\mathrm{~s} / \mathrm{s})}^{\mathrm{N}},
\end{aligned}
$$

where we have found it useful to regroup the terms so that

$$
T_{n}^{\mathrm{N}_{\mathrm{A}}}=T_{n(\mathrm{P} / \mathrm{S})}^{\mathrm{N}}+T_{n(\mathrm{P} / \mathrm{s})}^{\mathrm{N}}
$$

represents planetary wave interaction with synoptic and small scales,

$$
T_{n}^{\mathrm{N}_{\mathrm{B}}}=T_{n(\mathrm{~S} / \mathrm{S})}^{\mathrm{N}}
$$

represents all the interactions of synoptic waves, and

$T_{n}^{\mathrm{N}_{\mathrm{C}}}=T_{n(\mathrm{~S} / \mathrm{s})}^{\mathrm{N}}$

represents the interactions of synoptic waves with truly small-scale waves. Note that an analogous decomposition can be made of the enstrophy transfer $Q_{n}^{\mathrm{N}}$.

This scheme was implemented for both energy and enstrophy transfers by making the decomposition of eq. (36) in spectral space, transforming back into position space, and using the various interactions within each group and between groups in the non-linear terms of eq. (7). Transforming the results back to spectral space accomplishes the decomposition of eq. (37) and allows calculation of the terms in eq. (38).

\section{Kinetic energy spectra}

The kinetic energy of the total transient flow is summarized in Fig. 1a. The rotational kinetic energy for winter (summer) is given by the solid (short dashed) curves, while the divergent kinetic energy for winter (summer) is given by the dotted (dash-dotted) curves. The corresponding results for the kinetic energy of the mean flow are given in Fig. $1 \mathrm{~b}$ in the same form. All curves represent vertical integrals averaged over all years, and the $\log$ of the energy is plotted against the log of the wavenumber. The large-scale divergent transient kinetic energy is less than the rotational kinetic energy. For $n>10$, the rotational energy is at least an order of magnitude larger than the divergent energy. The entire transient divergent kinetic energy spectrum is dominated by the highfrequency component (not shown), suggesting the importance of the divergent circulation forced by latent heat release. The dominance of the transient spectrum by the rotational part for all but the largest scales motivates the focus on the rotational kinetic energy and enstrophy budgets.

The rotational mean flow spectrum (Fig. 1b) is remarkably similar in form to that presented by Boer and Shepherd (1983) for both winter and summer. The dominance of wavenumbers 1 and 3 (especially in winter) can be shown to be a result of the broad structure of the sub-tropical jet which in our (northern hemisphere reflected) case is symmetric about the equator. For wavenumbers $n>8$ the transient spectrum dominates the mean spectrum, in agreement with Boer and Shepherd (1983).

The distinct shift of the peak of the summer transient rotational spectrum to higher wavenumbers compared to winter (Fig. 1a), as well as the overall lower level of energy in summer are evidence of the seasonal dependence of the (northern hemisphere) general circulation. Specifically, in summer the mid-latitude baroclinic zone, characterized by strong mean vertical wind shear and meridional temperature gradient, is both weaker and shifted poleward compared to winter, resulting in a lower level of baroclinic generated eddy activity and a shift in spatial scale (White, 1982; Blackmon and White, 1982).

The frequency dependence of the transient rotational kinetic energy in winter is explored in Fig. 2a, which shows the total transient energy (solid curve), the annual cycle transient energy (the short dashed curve), the low-frequency energy transient energy (dotted curve) and the high-frequency transient energy (dash-dotted curve). By construction, the low- and high-frequency transient energies sum to the annual cycle transient energy (Section 2). The high-frequency energy dominates the low-frequency energy for the synoptic and small-scale waves, while the reverse is true for the planetary waves (except for $n=1)$.

The variability of the total transient energy is illustrated in Fig. 2b which compares the standard deviation $\sigma_{1}$ of the seasonal values of energy 

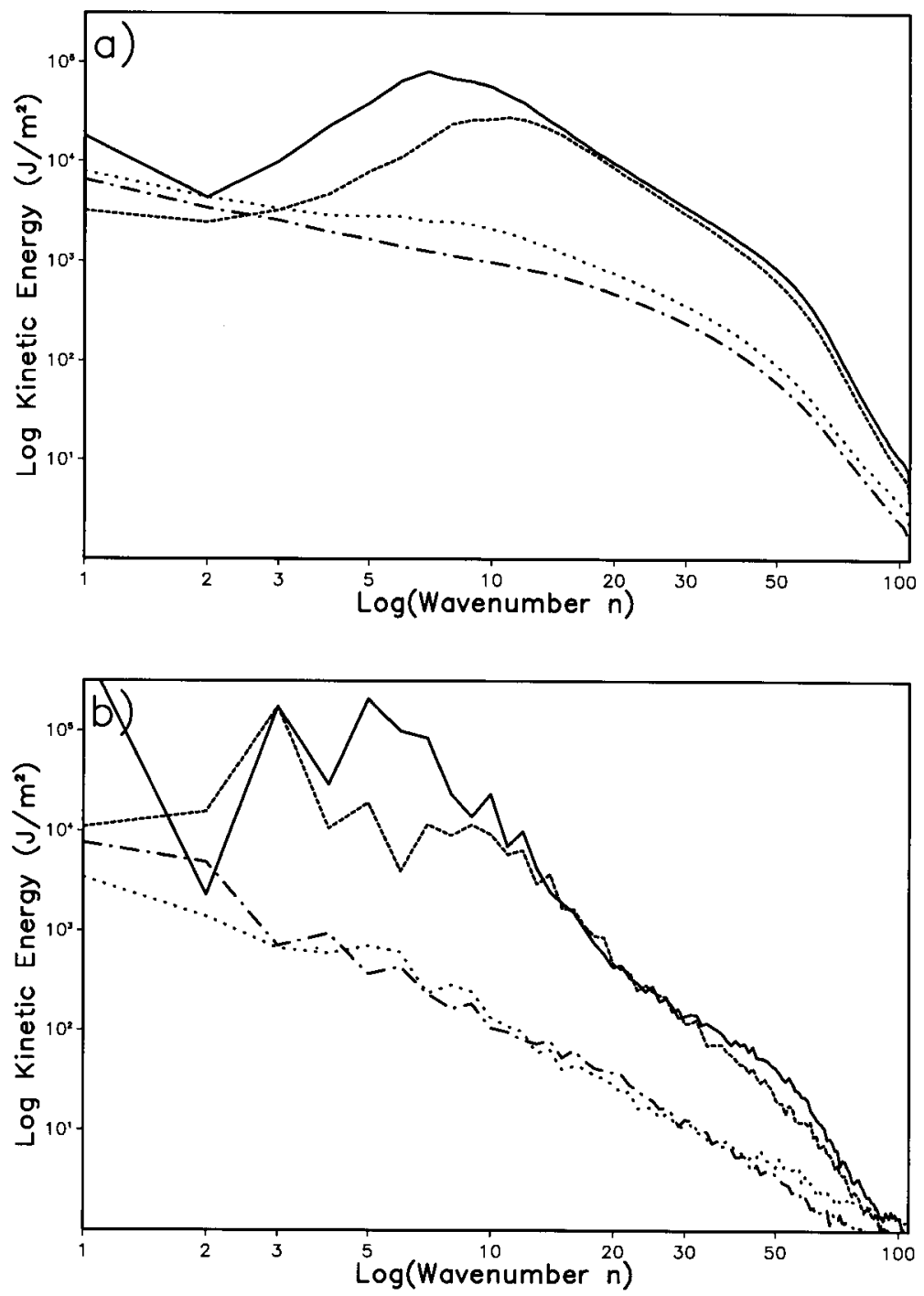

Fig. 1. (a) Log of transient vertically-integrated kinetic energy $\left(\mathrm{J} \mathrm{m}^{-2}\right)$ plotted versus $\log$ of wavenumber $n$. Average of 14 winters (15 summers) rotational kinetic energy given by solid (short dashed) curve. Average of 15 winters (summers) divergent kinetic energy given by dotted (dash-dotted) curve. (b) Log of vertically-integrated mean kinetic energy $\left(\mathrm{J} \mathrm{m}^{-2}\right)$ plotted versus $\log$ of wavenumber $n$. Otherwise as in (a).

(short-dash curve) and the standard deviation $\sigma_{2}$ of 10-day mean energies about the seasonal mean (dotted curve) with the total transient energy (solid curve). Near the peak of the spectrum $\sigma_{1}$ is about $15 \%$ of the total energy, and $\sigma_{2}$ is about $30 \%$. However, beyond the planetary scale range the uncertainty drops rapidly compared to the average energy.
The spectral slope $b$ is computed from the spectrum as a least-squares fit of the energy to the form $n^{-b}$ for 10 values of the wavenumber $n$ at a time, with the slope plotted as a function of the lowest wavenumber in the range. The results are shown in Fig. 3 for winter (solid curve) and summer (dashed curve), plotted against log of wavenumber. The thin curves indicate the uncer- 

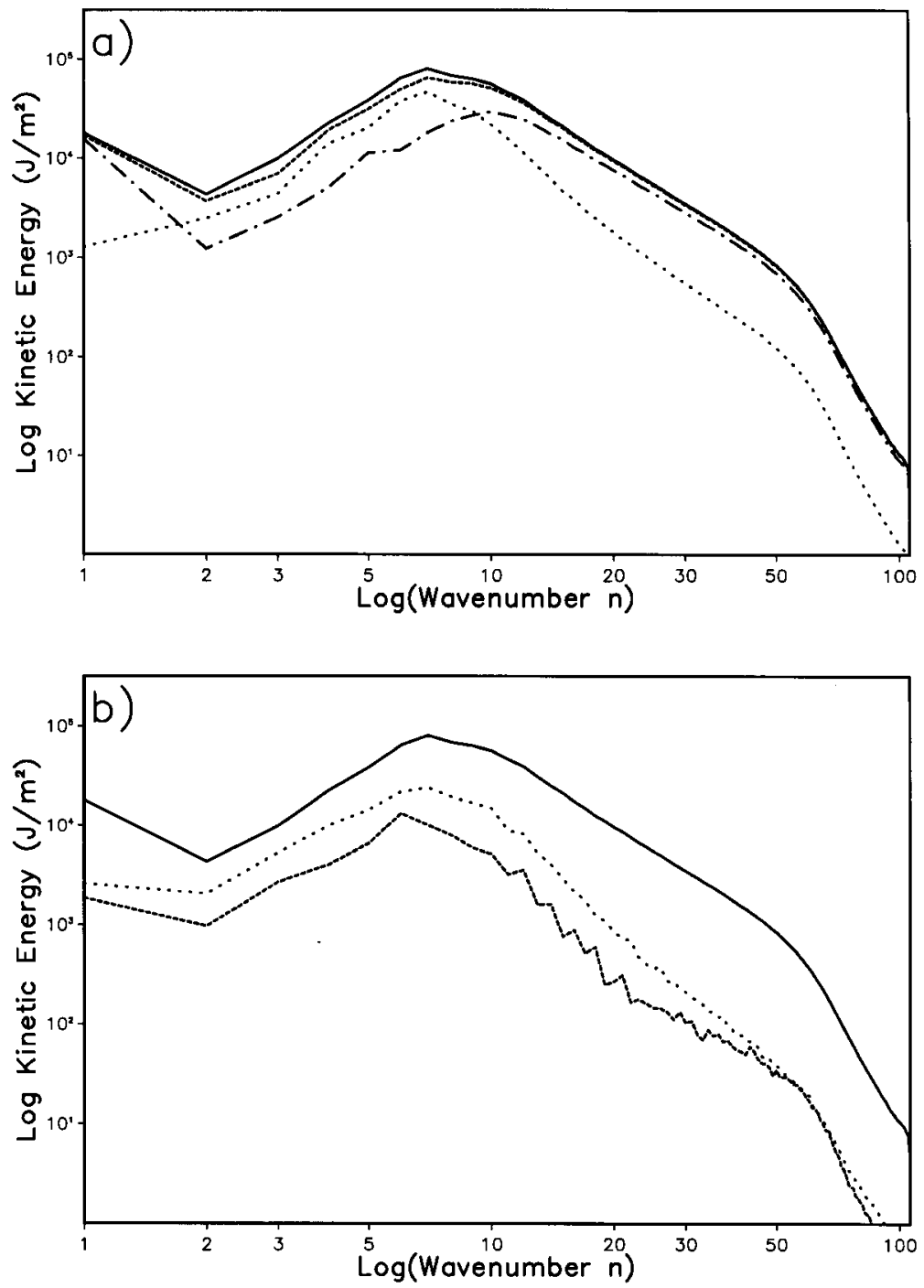

Fig. 2. (a) Log of vertically-integrated transient kinetic energy $\left(\mathrm{J} \mathrm{m}^{-2}\right)$ plotted versus log of wavenumber $n$. Average of 14 winters. Solid curve gives results from total transients, short dashed curve from transients about the annual cycle, dotted curve from low-frequency transients, and dash-dotted curve results from high-frequency transients. (b) Solid curve as in (a), short dashed curve gives log of interannual standard deviation of transient kinetic energy, dotted curve gives log of intraseasonal standard deviation, obtained from individual 10-day means.

tainty based on the interannual variability. In winter, $b$ lies consistently in the range of 2.5 to 2.7 over a broad range of wavenumbers (10 to 35$)$; this is true in summer over a slightly narrower range and for slightly larger values of $b$. The values for $b$ of 2.5 to 2.7 for the vertically-integrated winter energy are consistent with the results of Boer and Shepherd (1983), although the earlier results of Baer (1972) and Chen and Wiin-Nielsen (1978) suggest a slope nearer 3.0. The results in Fig. 3 for the spectral slope motivated the definitions of the wave groups given earlier.

The high wavenumber regime $(n>40)$ is characterized by a very steep drop-off in the spectrum 


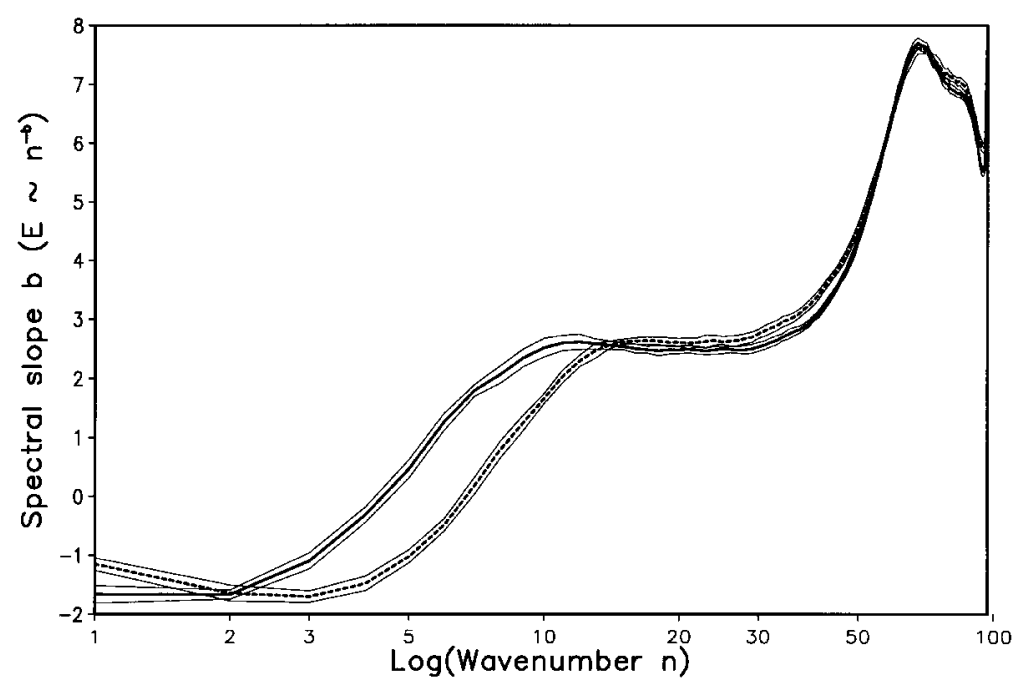

Fig. 3. Best fit slope $b$ of energy spectrum, from $E \sim n^{-b}$ as a function of $\log$ of wavenumber $n$. For each $n$ the fit was done over the range $n$ to $n+10$. Winter average (solid), summer average (short dashed). The interannual standard deviations are indicated by thin lines.

(rapidly increasing value of $b$ ), in agreement with the results of Trenberth and Solomon (1993). This is clearly inconsistent with the observations of $\mathrm{NG}$ and Högström et al., 1999, in which $b \sim 5 / 3$ in the mesocale range. Apparently the analysis/forecast system used in the reanalyses is heavily damped for these scales.

\section{Energy and enstrophy transfers}

\subsection{Energy transfers}

The total transient winter vertically-integrated energy balance (eq. (28)) is given in Fig. 4a. Here the non-linear term $T_{n}^{\mathrm{N}}$ (solid curve), the linear term $T_{n}^{\mathrm{L}}$ (short-dashed curve) and the source term $T_{n}^{\mathrm{S}}$ (dotted curve, obtained as a residual) are given. As with all the plots in this section, the total transient results are presented unless otherwise stated, and it is the wavenumber $n$ times the term $T$ that is plotted against $\log$ of wavenumber, ensuring that the area under the curve represents the total energy change. The source $T_{n}^{\mathrm{S}}$ agrees qualitatively with early estimates of Leith and Kraichnan (1972), and consists of baroclinic conversion (available potential to kinetic energy), dissipation, upward vertical flux of kinetic energy out of the $1000-100 \mathrm{hPa}$ layer considered and a term due to analysis increments (insertion of observational data in the forecast/analysis scheme). The very broad maximum extending over wavenumbers $n \sim 10-50$ is consistent with a baroclinic source, while the sink apparent at low $n$ is assumed to be due to friction*.

The linear term $T_{n}^{\mathrm{L}}$ represents barotropic interactions between the mean flow and the transients, and is linear only if the mean flow is considered fixed. The transient loss to the mean flow is greatest at $n=8$, while for larger scales there is a slight gain from the mean flow. The transient energy transfer given by the transient/transient interactions is from higher to lower wavenumbers, with the energy from a band centered on $n \sim 15$ being transferred to one centered on $n \sim 7$, the scale at which the energy peaks.

A summary of the mean flow kinetic energy

* The calculation of the separate components of the source term is beyond the scope of this study. It would have to be done using the precise formulation of the numerical forecast model used by ECMWF in the reanalyses, and also using the analysis increments due to data insertion. Its feasibility would depend upon what model data were stored and are available. We are not aware of any estimates of the wavenumber dependence of these quantities which would be of sufficient accuracy to be useful here. 

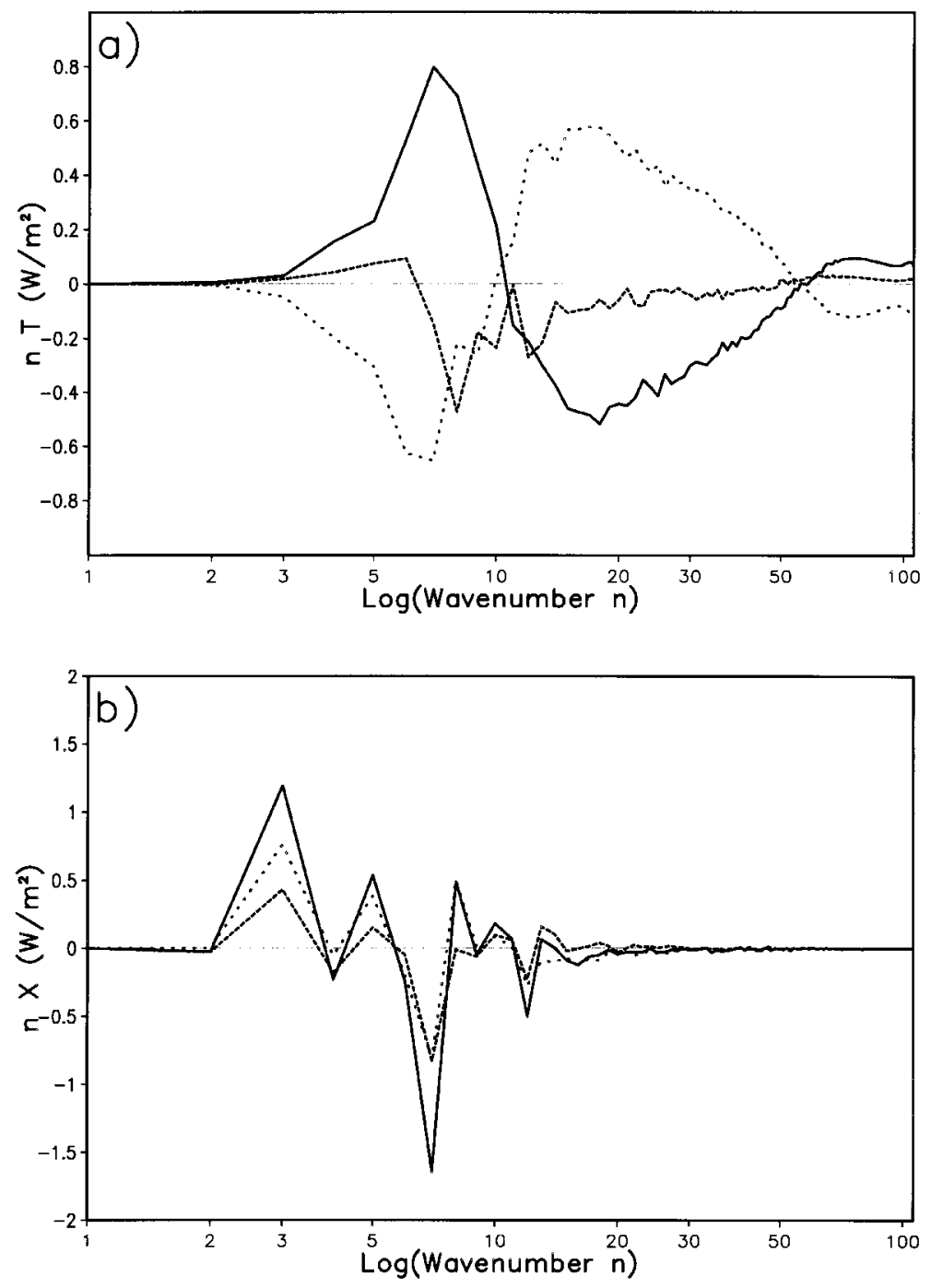

Fig. 4. (a) Winter vertically-integrated energy transfer terms for the total transient motion. The non-linear term $T^{\mathrm{N}}$, the linear term $T^{\mathrm{L}}$, and the source term $T^{\mathrm{S}}$ are given by the solid, short dashed, and dotted curves respectively. Units: $\left(\mathrm{W} \mathrm{m}^{-2}\right)$. The transfer terms are multiplied by wavenumber $n$, and plotted against $\log n$. (b) Verticallyintegrated energy transfer terms for the mean motion. The contribution to the non-linear term $X^{\mathrm{N}}$ due to mean flow interactions $X^{\mathrm{N}_{1}}$ is given by the short dashed curve, that due to transient flow feedback $X^{\mathrm{N}_{2}}$ by the dotted curve, and their sum by the solid curve. Otherwise as in (a).

budget (eq. (27)) is given in Fig. 4b. Here the linear term $X_{n}^{\mathrm{L}}$ (not shown) is truly linear and is very small; the mean budget is dominated by the mean/mean non-linear interactions $X_{n}^{\left(\mathrm{N}_{1}\right)}$ and the transient/transient interactions $X_{n}^{\left(\mathbf{N}_{2}\right)}$ whose sum is balanced by friction and analysis increments (see Section 3 for precise definitions). The mean/mean interaction term is shown as the short dashed curve, the transient/transient interaction term as the dotted curve, and their sum as the solid curve. The transient/transient term dominates, and transfers energy from $n=7$ to $n=3$, the latter related to the structure of the northern hemisphere jet as discussed above. 
In order to study the scale interactions in the term $T_{n}^{(\mathrm{N})}$ which are responsible for the upscale energy transfer, we show in Fig. 5a the total annual cycle $T_{n}^{(\mathrm{N})}$ (short-dashed curve) and the contribution to this term of interactions involving synoptic $(n=11-40)$ scales (solid curve)*. This set of interactions $T_{n}^{\mathrm{N}_{\mathrm{B}}}$, given in eq. (40), is responsible for most of the energy increase at scales smaller than $n \sim 10$. In fact these interactions tend to increase the energy also for smaller scales (up to $n \sim 15$ ), but between wavenumbers 10 and 15 are offset by the synoptic wave/planetary wave interactions (not shown). While the high-frequency transients contribute a modest amount to this positive tendency of large-scale energy, the major contribution to $T_{n}^{\mathrm{N}_{\mathrm{B}}}$ comes from the interactions between low and high frequencies (dotted curve). The interaction term is simply the difference between $T_{n}^{\mathrm{N}_{\mathrm{B}}}$ calculated with the annual cycle transients and the sum of $T_{n}^{\mathrm{N}_{\mathrm{B}}}$ calculated with low-frequency transients and (separately) with high-frequency transients. It contains all triple product terms which contain both high- and low-frequency components as well as wavenumbers within the synoptic range.

The appearance of zonal jets in quasi-geostrophic turbulence simulations (Panetta, 1993) raises the question of how much of the energy transfer seen in Fig. 4a, 5a results in energy creation at the zonal wavenumber $m=0$ component. Fig. $5 \mathrm{~b}$ compares the transient transfer $T_{n}^{\mathrm{N}}$ (now based on total transients) with the components representing non-linear transfer to the zonal flow ( $m=0$; short dashed curve) and to the very long zonal planetary waves $(m=1,2$; dot-dashed curve). Transfer to the zonal flow is seen to be very small, and that to $m=1$ and $m=2$ about $25 \%$ of the total.

However, the situation is very different with regard to the non-linear maintenance of the mean flow. Fig. 5c compares the transient/transient mean flow tendency $X_{n}^{\mathrm{N}_{2}}$ (solid curve) with the component yielding transfer only to $m=0$ (dashed curve). Also shown are the non-linear mean flow interaction term $X_{n}^{\mathrm{N}_{1}}$ (dotted curve) and the com-

* The results for the total transients and annual cycle transients are very similar in character, with the latter being slightly less intense. Since the low- and highfrequency transients are defined using departures from the annual cycle, they are best compared to the full set of annual cycle transients, and not the total transients. ponent giving transfer only to the $m=0$ flow (dash-dot curve). Clearly the energy transferred upscale by non-linear interactions in the mean flow predominantly ends up in the zonal flow. This upscale transfer is seen to lie entirely within the planetary scale range, with energy loss at $n=$ 7 and gain at $n=3$ and $n=5$. In the context of the zonal flow, the total wavenumber $n$ measures the meridional scale of the flow. The mean zonally symmetric jet structure in the atmosphere is maintained by non-linear terms only at the broadest meridional scales.

The variability of the upscale energy transfer $T_{n}^{\mathrm{N}}$ is explored in Fig. 6a, 6b. The solid line gives the total transient results, the short dashed lines define confidence levels based on $\sigma_{1}$, and the dotted lines confidence levels based on $\sigma_{2}$. The uncertainty of the total transient term $T_{n}^{\mathrm{N}}$ given in Fig. $6 \mathrm{a}$ is large. Approximately $16 \%$ of 10 -day periods yield an upscale energy tendency almost twice the mean, while an equal number show no upscale energy transfer whatever. However, if we limit the scale interactions to those involving synoptic scales only (term $T_{n}^{\mathrm{N}_{\mathrm{B}}}$ ), the variability is significantly smaller as shown in Fig. 6b. The upscale nature of the energy transfer from triads involving synoptic scales is fairly robust.

\subsection{Enstrophy transfers}

Fig. 7a gives the enstrophy (mean squared vorticity) of the total transients, annual cycle transients, and low- and high-frequency transients, multiplied by $a^{2}$. Here removing the annual cycle has little effect, while the low-frequency contribution is relatively small beyond $n \sim 15$. The non-linear term $Q_{n}^{\mathrm{N}}$ and the source term $Q_{n}^{\mathrm{S}}$ from the enstrophy budget eq. (31) are shown (multiplied by $a^{2}$ ) in Fig. 7b. The non-linear terms transfer enstrophy from a broad source regime $(n \sim 10-55)$ predominantly to small scales $(n>55)$. A small degree of upscale transfer is also noted, and shall be discussed later on. The linear term (not shown) is relatively quite small, so that the dominant balance is between non-linearity and the source term.

The planetary wave $(\mathrm{P})$ part of the non-linear enstrophy tendency $Q_{n}^{\mathrm{N}}$, defined in analogy to eq. (39), consists of separate sets of interactions between the $\mathrm{P}$ and synoptic wave (S) component, and between the $\mathrm{P}$ and the small-scale component (s). Each of these is plotted in Fig. 8a. The inter- 

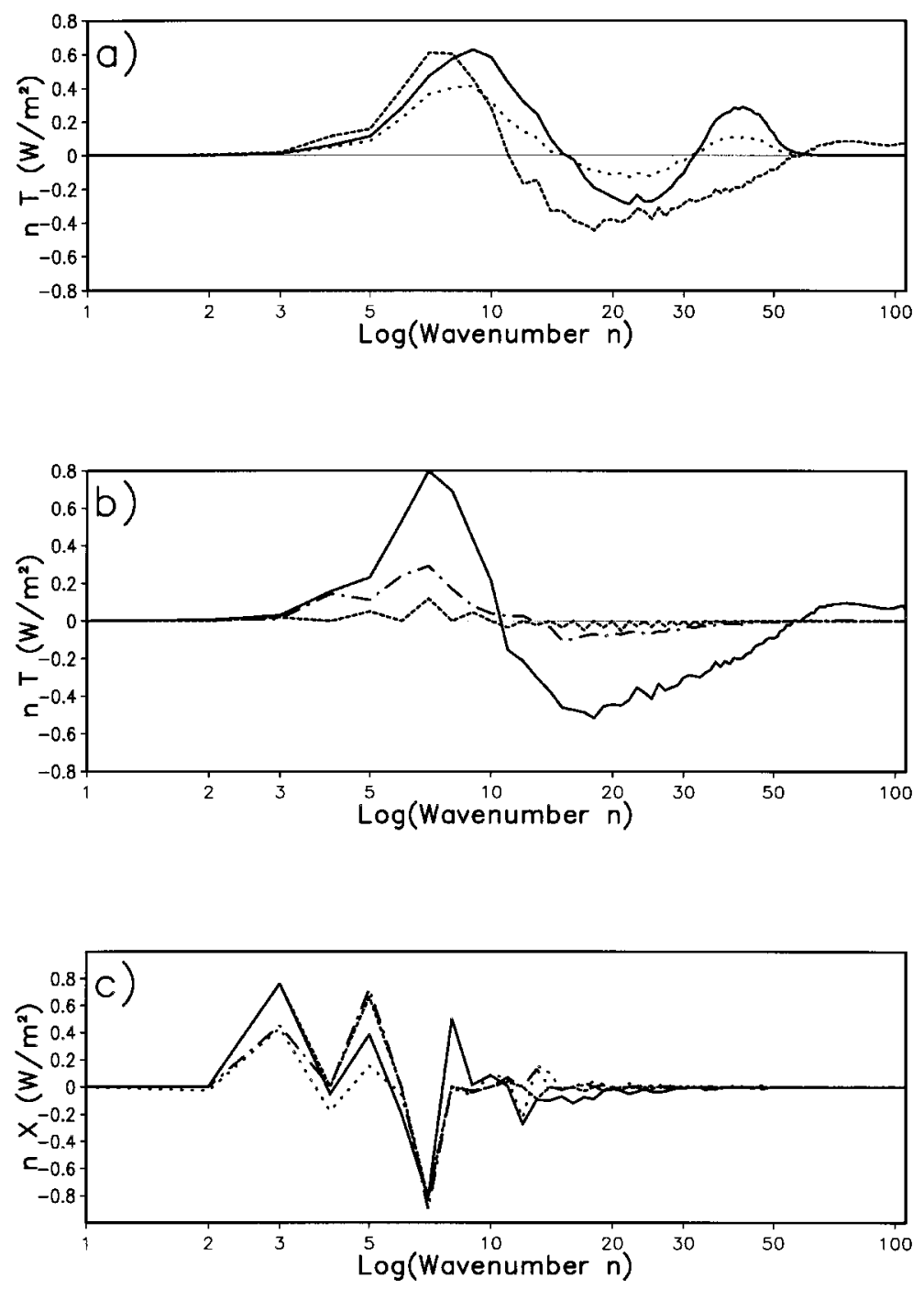

Fig. 5. (a) Winter vertically-integrated energy transfer terms. The non-linear term $T^{\mathrm{N}}$ based on the transient departure from the annual cycle is given by the short dashed line, the contribution to this term from interactions involving synoptic motions is given by the solid line, while the contribution to the synoptic interactions between low and high frequencies is given by the dotted curve. Units: $\left(\mathrm{W} \mathrm{m}^{-2}\right)$. The transfer terms are multiplied by wavenumber $n$, and plotted against $\log n$. (b) The non-linear term $T^{\mathrm{N}}$ based on the total transient motion is given by the solid curve, the part yielding energy to zonal wavenumber $m=0$ is given by the short dashed curve, and the contribution to $T^{\mathrm{N}}$ from $m=1$ and $m=2$ by the dash-dotted curve. Otherwise as in (a). (c) The non-linear term $X^{N_{2}}$ giving the transient/ transient feedback onto the mean flow is given by the solid curve. The mean/mean non-linear term $X^{\mathrm{N}_{1}}$ is given by the dotted curve. The components of these terms corresponding to changes in the mean zonal flow $(m=0)$ are given by the dashed and dot-dashed curves for the transient/transient and mean/mean interactions, respectively. 

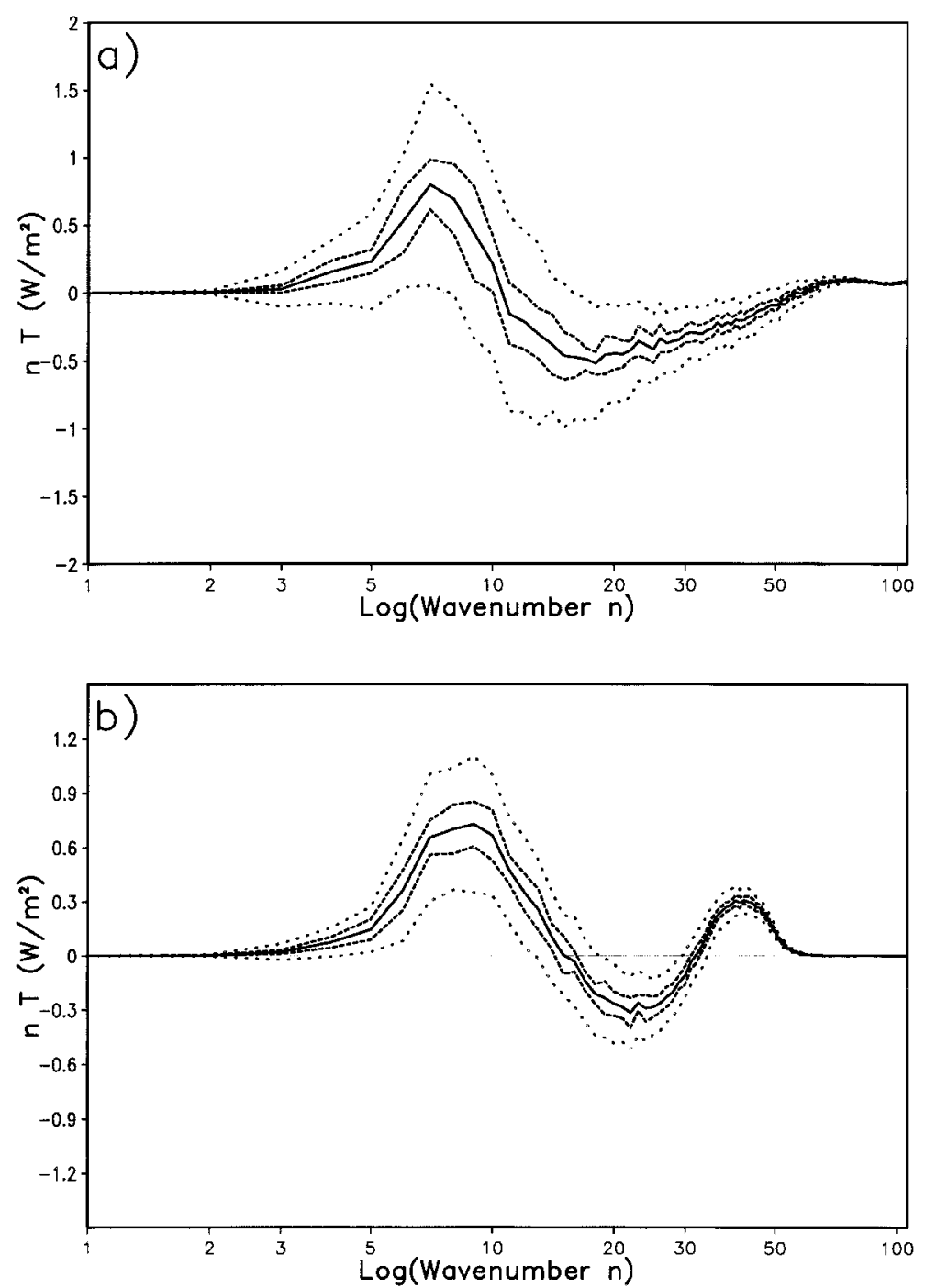

Fig. 6. (a) Winter vertically-integrated energy transfer term for the total transient motion $T^{\mathrm{N}}$ (solid line). The short dashed curves indicate one standard deviation departure based on variations between winters, the dotted curves one standard deviation departure based on variations within a season. Units: $\left(\mathrm{W} \mathrm{m}^{-2}\right)$. The transfer terms are multiplied by wavenumber $n$, and plotted against $\log n$. (b) As in (a), but for the contribution to $T^{\mathrm{N}}$ due to interactions involving synoptic waves.

action between $\mathrm{P}$ and the larger scale synoptic waves $11 \leqslant n \leqslant 20$ is given by the long-dash curve, the interaction between $\mathrm{P}$ and the smaller scale synoptic waves $21 \leqslant n \leqslant 40$ is given by the shortdash curve, and the interaction between the $\mathrm{P}$ and the small-scale waves $41 \leqslant n \leqslant 106$ by the dashdotted curve. The figure also shows the sum of all three terms (plus the small $\mathrm{P}$ self-interactions).
The three sets of interactions form a chain: $\mathrm{P} /$ larger scale $\mathrm{S}$ interactions remove enstrophy from $n \sim 5-15$ and transfer it to the range $n \sim 15-30$, from which $\mathrm{P} /$ smaller scale $\mathrm{S}$ interactions transfer the enstrophy further downscale to $n \sim 30-50$. The $\mathrm{P} /$ small-scale interactions take the enstrophy in the latter range and transfer to $n>50$, this final transfer accounting for about 

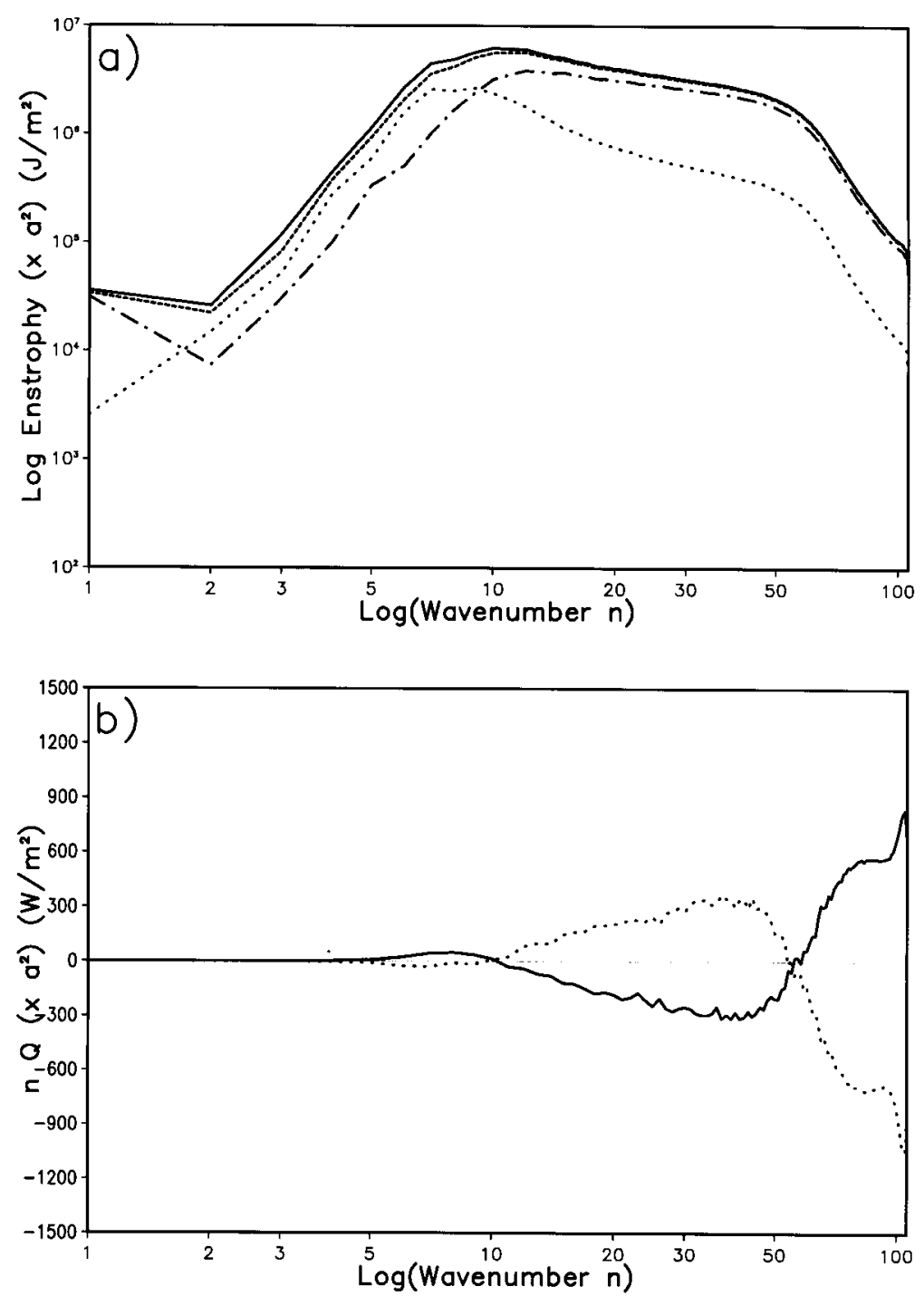

Fig. 7. (a) $\log$ of winter vertically-integrated enstrophy ( $\times a^{2}$, where $a$ is the earth's radius), plotted against $\log$ of wavenumber $n$. Units: $\left(\mathrm{J} \mathrm{m}^{-2}\right)$. Solid curve gives total transient result, short dashed curve is from transient departure from annual cycle, dotted curve from low-frequency fluctuations, dash-dotted curve from high-frequency fluctuations. (b) Winter vertically-integrated enstrophy transfer term $a^{2} Q^{\mathrm{N}}$ (solid curve). Dotted curve gives enstrophy source term $a^{2} Q^{\mathrm{S}}$. Units: $\left(\mathrm{W} \mathrm{m}^{-2}\right)$. The transfer and source terms are multiplied by wavenumber $n$, and plotted against $\log n$.

$30 \%$ of the total small-scale enstrophy tendency shown in Fig. 8b. The transfers depicted in Fig. 8a are consistent both with simple advection of enstrophy by the longest scales (Lorenz, 1969) and with the dynamics described by Kraichnan (1971).

The behavior of these large $\mathrm{P}$ wave transfers can be contrasted to the more local transfers within the synoptic scales and between the synoptic and smaller scales, analogous to the energy transfers given in eqs. (40) and (41). These are given in Fig. 8b. The interactions between larger and smaller synoptic scales transfer enstrophy out of the larger scale portion $(n \sim 15-32)$ of the 

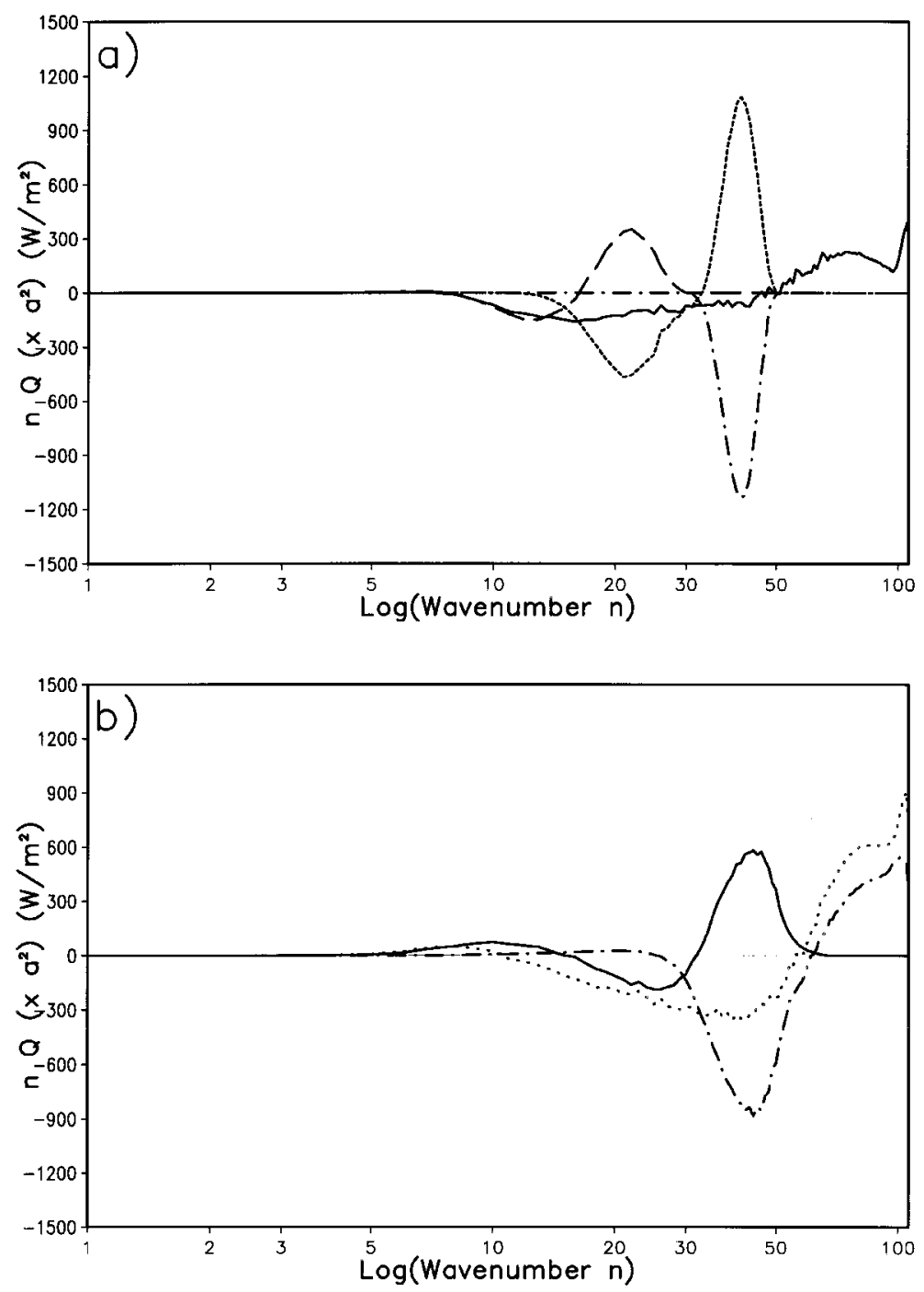

Fig. 8. (a) Contributions to the vertically-integrated winter total transient enstrophy non-linear transfer term $a^{2} Q^{\mathrm{N}}$. The total contribution involving planetary waves is given by the solid curve, the contribution from planetary/larger synoptic wave $(11 \leqslant n \leqslant 20)$ interactions by the long-dash curve, the contribution from planetary/smaller synoptic wave $(21 \leqslant n \leqslant 40)$ interactions by the short-dash curve, and the contribution from planetary/small-scale interactions by the dash-dotted curve. Units: $\left(\mathrm{W} \mathrm{m}^{-2}\right)$. The transfer terms are multiplied by wavenumber $n$, and plotted against $\log n$. (b) Contribution to $a^{2} Q^{\mathrm{N}}$ from interactions between synoptic scales is give by the solid curve, the contribution due to smaller synoptic/small-scale interactions by the dash-dotted curve. The total $a^{2} Q^{\mathrm{N}}$ is given by the dotted line. Otherwise as in (a).

overall source of Fig. $7 \mathrm{~b}$, with both strong downscale and weak upscale transfer apparent. The increase in enstrophy in the range $n \sim 33-55$ due to this transfer is offset by the interactions between smaller synoptic scales and small scales which transfer enstrophy to $n>60$, providing about $2 / 3$ of the total enstrophy tendency there. These relatively local interactions are dominated by highfrequency transients (not shown), in contrast to the upscale energy transfer, for which interactions

Tellus 51A (1999), 5 
between high and low frequencies dominate. It is noteworthy that the non-local (advective) interactions given in Fig. 8a play a much more important rôle in the enstrophy budget than in the energy budget.

\subsection{Seasonal dependence}

The seasonal dependence of the basic kinetic energy balance is similar to that of the kinetic energy spectrum. Fig. 9a shows that the summer non-linear tendency peak is half the winter peak, and is shifted to slightly higher $n$. These comments also apply to the total kinetic energy source $T_{n}^{\mathrm{S}}$. As discussed in the previous section, the decrease in magnitude and scale of the energy transfers is related to the seasonal change in the general circulation. The dominance of the large positive non-linear tendency at large scales by interactions involving synoptic scales, and the importance of interactions between low and high frequencies are also seen in the summer results (not shown). As in winter, the transient zonal flow $(m=0)$ is not strongly forced by the non-linear tendencies, while the forcing of the planetary waves $(m=1,2)$ is about $1 / 4$ of the total tendency. Finally, the varia- bility of the non-linear tendency for summer reproduces the main conclusions from winter: the synoptic non-linear tendency shows less variability than the tendency calculated with all terms, particularly for the large scales.

Because the enstrophy balance weights higher wavenumbers, there is less of a seasonal dependence as depicted in Fig. 9b. The enstrophy source $Q_{n}^{\mathrm{S}}$ is nearly as great in summer as in winter. This is also true of the non-linear transfer out of the source region to small scales. Only the (much weaker) transfer to larger scales shows a summer increase in the transition wavenumber. The overall properties of the separate components of the nonlinear enstrophy transfer (interactions among different wave groups) are remarkably similar between summer (not shown) and winter, the main difference being the summertime overall weakness of the planetary wave interactions.

\subsection{Energy and enstrophy fluxes}

For a truly inertial sub-range of wavenumbers, the forcing and dissipation (source term) vanishes and hence in equilibrium the energy tendency must also (Batchelor, 1953). In this picture the

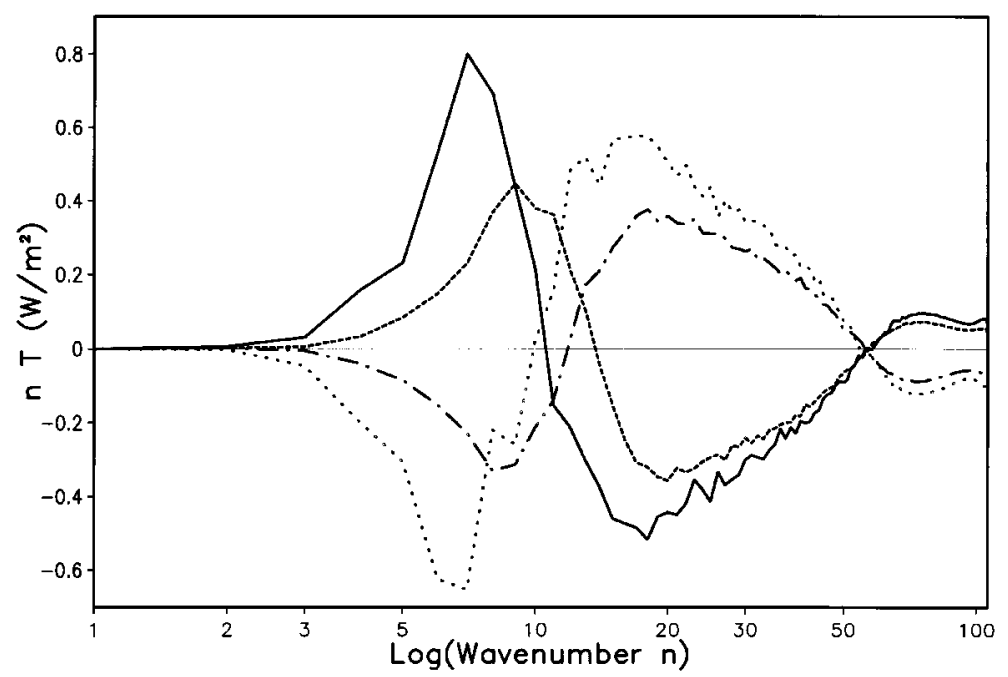

Fig. 9. (a) Vertically-integrated energy tendency terms $T^{\mathrm{N}}$ and $T^{\mathrm{S}}$, based on total transients. Solid curve is $T^{\mathrm{N}}$ for winter, dotted curve is $T^{\mathrm{S}}$ for winter, short-dash curve is $T^{\mathrm{N}}$ for summer, and dot-dash curve is $T^{\mathrm{S}}$ for summer. Units: $\left(\mathrm{W} \mathrm{m}^{-2}\right)$. The transfer terms are multiplied by wavenumber $n$, and plotted against $\log n$. (b) Verticallyintegrated enstrophy tendency terms $a^{2} Q^{\mathrm{N}}$ and $a^{2} Q^{\mathrm{S}}$ based on total transients. Solid curve is $a^{2} Q^{\mathrm{N}}$ for winter, dotted curve is $a^{2} Q^{\mathrm{S}}$ for winter, short-dash curve is $a^{2} Q^{\mathrm{N}}$ for summer, and dot-dash curve is $a^{2} Q^{\mathrm{S}}$ for summer. Otherwise as in (a). 
flux of energy or enstrophy is constant, and is termed a cascade. The energy and enstrophy fluxes, calculated according to eqs. (32) and (33) in Section 3, are given in Figs. 10a,b. While the negative energy flux denoting upscale energy transfer and the positive enstrophy flux denoting downscale transfer are expected on the basis of 2-dimensional turbulence arguments, there is no range of wavenumbers over which either flux is even approximately constant. Futhermore, there is a broad range $(n \sim 20-40)$ in which both fluxes are non-zero. In agreement with previous studies, we find that the available wavenumber range between the forcing due to baroclinic energy conversion (at $n \sim 10-20$ ) and the largest scales is small, precluding a true upscale energy cascade. For the enstrophy flux, the broad range of enstrophy dissipation seen in Fig. $7 b$ (inconsistent
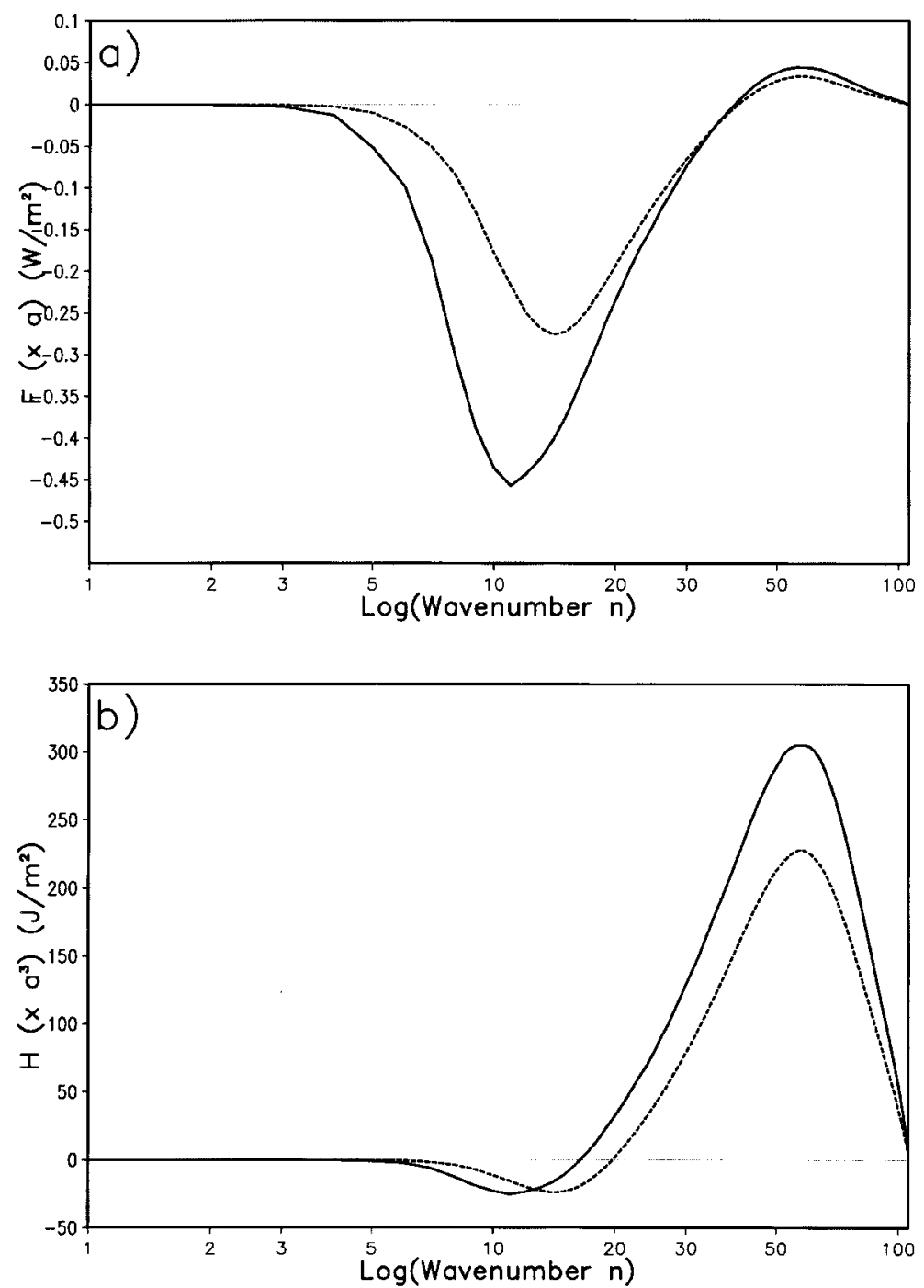

Fig. 10. (a) Energy flux $a F$ based on vertically-integrated winter (solid) and summer (short-dash) total transient nonlinear tendencies plotted against $\log$ of wavenumber $n$. See text for details. Units: $\left(\mathrm{W} \mathrm{m}^{-2}\right)$. (b) As in (a), but for enstrophy flux $\mathrm{a}^{3} \mathrm{H}$.

Tellus 51A (1999), 5 
with the nearly inviscid limit of classical turbulence theory) inhibits a true enstrophy cascade.

\subsection{Time scales}

The energy balance depicted in Fig. 9 indicates two basic regimes: a planetary scale regime $(n=$ $0-10)$ in which the non-linear tendency maintains the flow against dissipation, and an intermediate scale regime in which the net baroclinic conversion (surplus of baroclinic conversion over dissipation) maintains the energy against non-linear transfer. Consider the energy contained in planetary scales, large synoptic scales $(11 \leqslant n \leqslant 20)$ and small synoptic scales $(21 \leqslant n \leqslant 40)$, labeling them $E_{\mathrm{P}}, E_{\mathrm{S}}$ and $E_{\mathrm{SS}}$ respectively, and consider the processes that maintain them at these scales, namely nonlinear transfer $\left(N_{\mathrm{P}}\right)$, and net conversion $\left(C_{\mathrm{S}}\right.$ and $C_{\mathrm{SS}}$ ) respectively. (These are defined formally in Section 9). From each energy and maintenance component we derive a corresponding time scale: $\tau_{\mathrm{P}}=E_{\mathrm{P}} / N_{\mathrm{P}}, \quad \tau_{\mathrm{S}}=E_{\mathrm{S}} / C_{\mathrm{S}}$ and $\tau_{\mathrm{SS}}=E_{\mathrm{SS}} / C_{\mathrm{SS}}$. Each time scale is simply the time that would be required for the source to replenish the corresponding energy. For summer, we shift the definitions of the regimes slightly to take into account the shift in overall energy balance to smaller scales as in Fig. 9a: $n=0-13$ (planetary), $n=14-23$ (large synoptic) and $n=24-43$ (small synoptic). The results for winter and summer characteristic time scales are given in Table 1.

That $\tau_{\mathrm{P}}$ shows so little difference between winter and summer in spite of large differences in the energy indicates the relative strength of the nonlinear terms is about the same in each season. Further, the net baroclinic conversion into synoptic scales is of roughly the same strength compared to the appropriate kinetic energy as is the nonlinear tendency. It is only in the smaller synoptic scales that the relative magnitude of the net baroclinic conversion increases dramatically.

Table 1. Characteristic time scales (days) for the planetary, large synoptic and small synoptic waves in winter and summer

\begin{tabular}{lccc}
\hline Season & $\tau_{\mathrm{P}}$ & $\tau_{\mathrm{S}}$ & $\tau_{\mathrm{sS}}$ \\
\hline winter & 9.4 & 8.0 & 3.8 \\
summer & 8.5 & 9.4 & 4.0 \\
\hline
\end{tabular}

\section{Summary}

We have the following general conclusions based on the reanalyses:

(1) The spectral slope of kinetic energy in the range $n \sim 10-40$ is roughly $-2.5 \sim-2.7$. Based on the variability of the slope on seasonal and 10 -day time scales, this slope is significantly different than the classical turbulence theory prediction of -3 . Further discussion of this point is given in the Discussion section.

(2) There is no indication of the $-5 / 3$ mesoscale energy regime seen in observations, in agreement with previous work (Trenberth and Solomon, 1993).

(3) The broad distribution of enstrophy dissipation for these scales is far from the nearly inviscid limit in which dissipation only occurs at the highest wavenumbers.

(4) The non-linear energy flux is upscale, and the non-linear enstrophy flux is downscale, but a true cascade is not seen in either case. The lack of available wavenumber space between the baroclinic forcing and the planetary scale precludes the upscale energy cascade, while the lack of enstrophy cascade is consistent with point (3) and the lack of available wavenumber space between the baroclinic forcing and the dissipation.

(5) The (positive) non-linear transient tendency at large scales is quite variable, with very roughly $16 \%$ of 10 -day periods yielding a tendency twice the mean, and $16 \%$ showing no upscale tendency whatever. This variability is greatly reduced when only triads involving synoptic scales are retained. Much of the energy transferred by these triads involves interactions between high- and low-frequency components of the flow. The planetary scale energy supported by these transients is predominantly in the non-zonal $m \neq 0$ flow. However, the mean zonal flow $m=0$ is supported by nonlinear transient/transient and mean/mean interactions for the largest meridional scales $(n=3$ and $n=7$ ), but loses energy to these interactions for $n=7$.

(6) Non-local planetary wave advection of smaller scales plays an important rôle in the downscale transfer of enstrophy, being responsible for about $1 / 3$ of the total tendency at very small scales in winter. More local interactions also play a rôle in the enstrophy transfer. 
(7) The main seasonal effects are a dramatic weakening in summer of the total energy and shifting to higher wavenumber of the peak energy, and a distinct shift to higher scales in the transition between the large-scale and synoptic-scale regimes in the energy budget.

(8) The time scale of non-linear maintenance of planetary waves is roughly the same ( $\sim 9$ days) as that of baroclinic maintenance of the larger synoptic waves. The baroclinic support of the smaller synoptic scales is associated with a more rapid time scale ( $\sim 4$ days).

\section{Discussion}

The finding that the upscale energy transfer is not only dominated by synoptic scale interactions, but also by interactions between fluctuations with periods of $\sim 1-9$ and 11-90 days helps to interpret the turbulence diagnostics in the context of meteorological phenomena. In particular it indicates the importance of highly structured flows in which higher frequency smaller synoptic scales strongly interact with lower frequency, larger scales. Blocking is one example which may be of importance during the winter season (Nakamura and Wallace, 1993). The circulation supported by these interactions is predominantly non-zonal in nature. The upscale energy transfer of synoptic interactions is highly robust, for it occurs in a great majority of individual 10-day periods. Furthermore, circulations with these scales should be well represented by the reanalyses, suggesting that this source of large-scale variability is fundamental to the atmosphere.

A synthesis of our results relating to the classical turbulence theory is presented in Fig. 11, which shows the energy flux $F_{n}$, enstrophy flux $H_{n}$, energy source $T_{n}^{\mathrm{s}}$, and the spectral slope $b$, all scaled to fit on one plot. Enstrophy is transferred downscale, while energy is transferred upscale, with a resulting approximately -2.5 energy spectrum. The source term, determined to a large extent by the flow itself, encompasses a very broad range of wavenumbers and in fact is largest at scales for which the energy flux is largest. The degree to which this picture can be interpreted in terms of the classical scaling theory (Orszag, 1977), which assumes a localized source and a very wide range of wavenumbers both above and below that of the source, is severely limited. The weak downscale energy cascade accompanying the dominant enstrophy cascade seen in Fig. 11, as well as the weak upscale enstrophy cascade accompanying the dominant energy cascade can be understood as corrections due to the large scale nature of the source and the narrow range of available smaller

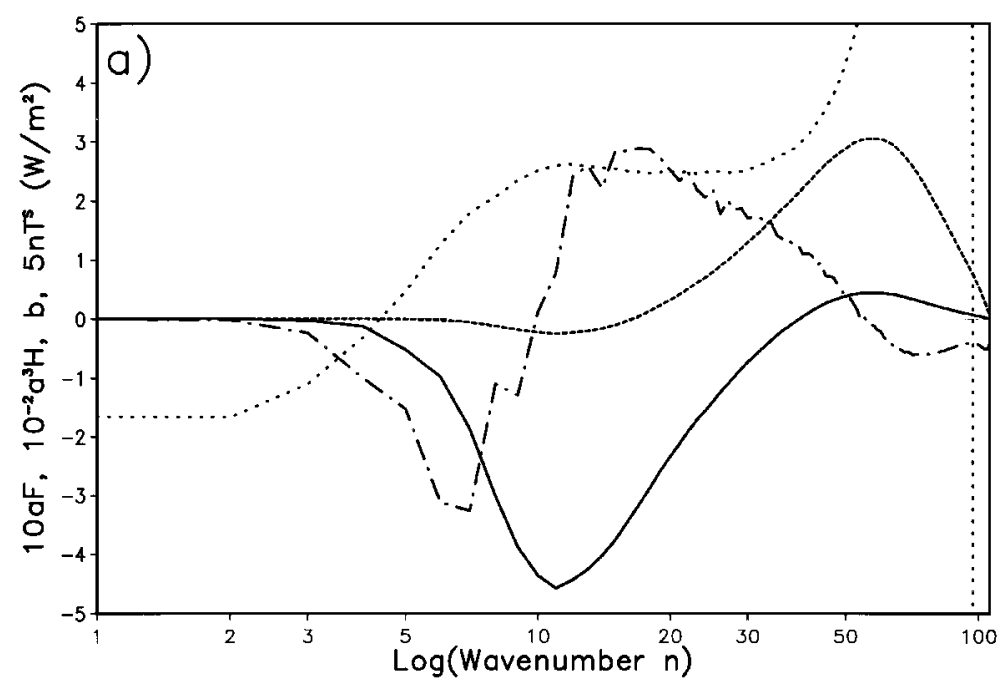

Fig. 11. Composite plot of winter vertically-integrated energy flux $F$, enstrophy flux $H$, energy source $T^{\mathrm{S}}$ and bestfit spectral slope $b$. Solid curve is $10 a F$, short-dashed curve is $10^{-2} a^{3} H$, dash-dotted curve is $5 n T^{\mathrm{s}}$, and dotted curve is slope $b$. Units: $\left(\mathrm{W} \mathrm{m}^{-2}\right)$ for all curves but $b$, which is dimensionless.

Tellus 51A (1999), 5 
wavenumbers (Terry and Newman, 1998). The effects of having a source which is determined by the flow itself and which has a very wide spectral extent are hard to understand (Panetta, 1993) in terms of simple scaling. A more comprehensive theory taking into account the nature of the interactive baroclinic source term is needed.

The energy spectrum falls off with an exponent of about -2.5 in the energy cascading range $(n \sim 10-40)$, and thereafter falls off much more steeply. The -2.5 slope is not necessarily incompatible with previous observational studies which do not define a single exponent clearly. However, it is incompatible with turbulence theories, although the very broad energy source $T_{n}^{\mathrm{S}}$ is consistent (intuitively speaking) with an energy spectrum that falls off less quickly with wavenumber $n$ than the classical -3 spectrum. Very weakly forced turbulent flows tend to produce spectra much steeper than -3 , associated with isolated coherent vortices and a great deal of intermittency (Basdevant, et al., 1981; McWilliams and Chow, 1985). Charney (1971) points out that a -4 spectrum is predicted for a series of discontinuities. Non-local interactions give only very weak logarithmic corrections to the -3 spectrum (Kraichnan, 1971). Nor are rotating dishpan experiments helpful, as they give a very wide range of spectra depending on the forcing (see Morita and Uryu (1989) for recent results). It is noteworthy that a spectrum characterized by a $-8 / 3$ slope (which is close to our results) is predicted by semi-geostrophic theory in association with the formation of fronts (Andrews and Hoskins, 1978). While it is not clear physically that the presence of strong fronts would dominate the large-scale spectrum, they are likely to play a rôle at subsynoptic scales.

The rapid steepening of the spectrum in the enstrophy cascading regime $n>40$ is highly inconsistent with the observational results of NG which clearly indicate a continuous flattening as the scale is decreased. Along with the broad region of enstrophy dissipation seen at these wavenumbers, this evidence strongly suggests that the numerical model and data assimilation scheme incorporate excessive damping for scales smaller than $n \sim 40$.

The diagnosis of the barotropic mode in this paper, while it includes the vertically averaged effects of higher baroclinic modes, is but a first step in the more complete non-linear analysis.
Analysis of the higher vertical (baroclinic) modes is not expected to fundamentally change the upscale energy transfer picture, but will paint a richer picture of the baroclinic (mode to mode) conversion, as well as the downscale enstrophy transfer.

\section{Acknowledgements}

We are grateful for the encouragement and helpful advice of A. Wiin-Nielsen, E. Källén, D. Newman, T. DelSole and advice of anonymous referees. One of us (DMS) was supported by the National Science Foundation under grant ATM-9321354, the National Aeronautics and Space Administration under grant NAG5-4977, and the National Oceanic and Atmospheric Administration under grant NA76-GP0258.

\section{Appendix}

We here define various terms used in calculating Table 1 . The planetary wave energy, large synoptic wave energy and small synoptic wave energy are given by:

$$
\begin{aligned}
& E_{\mathrm{P}}=\sum_{n=0}^{n=p} E_{n}^{\prime}, \\
& E_{\mathrm{S}}=\sum_{n=p+1}^{n=s} E_{n}^{\prime}, \\
& E_{\mathrm{SS}}=\sum_{n=s+1}^{n=t} E_{n}^{\prime},
\end{aligned}
$$

where $p, s$ and $t$ are integers which have the values 10, 20 and 40 for winter (respectively) and 13, 23 and 43 for summer.

The planetary wave non-linear tendency $N_{\mathrm{p}}$ is given by

$N_{\mathrm{P}}=\sum_{n=0}^{n=p} T_{n}^{\mathrm{N}}$,

whereas the large and small synoptic wave conversion tendencies $C_{\mathrm{s}}$ and $C_{\mathrm{ss}}$ are given by

$C_{\mathrm{S}}=\sum_{n=p+1}^{n=s} T_{n}^{\mathrm{S}}$,
$C_{\mathrm{SS}}=\sum_{n=s+1}^{n=t} T_{n}^{\mathrm{S}}$. 


\section{REFERENCES}

Andrews, D. G. and Hoskins, B. J. 1978. Energy spectra predicted by semi-geostrophic theories of frontogenesis. J. Atmos. Sci. 35, 509-512.

Baer, F. 1972. An alternate scale representation of atmospheric energy spectra. J. Atmos. Sci. 29, 649-664.

Bartello, P. 1995. Geostrophic adjustments and inverse cascades in rotating stratified turbulence. J. Atmos. Sci. 52, 4410-4428.

Basdevant, C., Legras, B. and Sadourny, R. 1981. A study of barotropic model flows: intermittency, waves and predictability. J. Atmos. Sci. 38, 2305-2326.

Batchelor, G. K. 1953. The theory of homogeneous turbulence. Cambridge University Press, $197 \mathrm{pp}$.

Blackmon, M. L. and White, G. H. 1982. Zonal wavenumber characteristics of Northern Hemisphere transient eddies. J. Atmos. Sci. 39, 1985-1998.

Boer, G. J. 1994. Mean and transient spectral energy and enstrophy budgets. J. Atmos. Sci. 51, 1765-1779.

Boer, G. J. and Shepherd, T. G. 1983. Large-scale two-dimensional turbulence in the atmosphere J. Atmos. Sci. 40, 164-184.

Charney, J. G. 1971. Geostrophic turbulence. J. Atmos Sci. 28, 1087-1095.

Chen, T.-C. and Wiin-Nielsen, A. 1978. Nonlinear cascades of atmospheric energy and enstrophy. Tellus 28, 313-322.

Fjørtoft, R. 1953. On the changes in the spectral distribution of kinetic energy in two-dimensional, nondivergent flow. Tellus 5, 225-230.

Gage, K. S. and Nastrom, G. D. 1986. Theoretical interpretation of atmospheric wavenumber spectra of wind and temperature observed by commercial aircraft during GASP. J. Atmos. Sci. 43, 729-740.

Gibson, J. K., Källberg, P., Uppala, S., Hernandez, A., Nomura, A. and Serrano, E. 1997. ECMWF re-analysis project report series, vol. I. ERA Description. European Centre for Medium-Range Weather Forecasts, Reading, UK. 72 pp.

Gifford, F. A. 1988. A similarity theory of the tropospheric turbulence energy spectrum. J. Atmos. Sci. $\mathbf{4 5}$ 1370-1379.

Haidvogel, D. B. and Held, I. M. 1980. Homogeneous quasi-geostrophic turbulence driven by a uniform temperature gradient. J. Atmos. Sci. 37, 2644-2660.

Högström, U., Smedman, A.-S. and Bergstrom, H. 1999. A case study of two-dimensional turbulence. Atmos. Sci. 56, 959-976.

Holloway, G. 1983. Effects of planetary wave propagation and finite depth on the predictability of atmospheres. J. Atmos. Sci. 40, 314-327.

Horn, L. H., and Bryson, R. A. 1963. An analysis of the geostrophic kinetic energy spectrum of large-scale atmospheric turbulence. J. Geophys. Res. 68, 1059-1064.

Hoskins, B. J. 1983. Modelling of the transient eddies and their feedback on the mean flow. In: Large-scale dynamical processes in the atmosphere (eds. Hoskins, B. J. and Pearce, R. P.). Academic Press, pp. 169-199.

Hoskins, B. J., James, I. N. and White, G. H. 1983. The shape, propagation and mean-flow interaction of large-scale weather systems. J. Atmos. Sci. 40, $1595-1612$.

Hoyer, J.-M. and Sadourny, R. 1982. Closure modeling of fully developed baroclinic instability. J. Atmos. Sci. 39, 707-721.

Hua, B. L., and Haidvogel, D. B. 1986. Numerical simulations of the vertical structure of quasi-geostrophic turbulence. J. Atmos. Sci. 43, 2923-2936.

Julian, P. R., Washington, W. M., Hembree, L. and Ridley, C. 1970. On the spectral distribution of largescale atmospheric kinetic energy. J. Atmos. Sci. 27, 376-387.

Kao, S.-K. and Wendell, L. I. 1970. The kinetic energy of the large-scale atmospheric motion in wavenumberfrequency space. J. Atmos. Sci. 27, 359-374.

Koshyk, J. N. and Boer, G. J. 1995. Parameterization of dynamical subgrid-scale processes in a spectral GCM. J. Atmos. Sci. 52, 965-975.

Kraichnan, R. H. 1967. Inertial ranges in two-dimensional turbulence. Phys. Fluids 10, 1417-1423.

Kraichnan, R. H. 1971. Inertial-range transfer in twoand three-dimensional turbulence. J. Fluid Mech. 47 , 525-535.

Lander, J. and Hoskins, B. J. 1997. Believable scales and parameterizations in a spectral model. Mon. Wea. Rev. 125, 292-303.

Lambert, S. J. 1981. A diagnostic study of global energy and enstrophy fluxes and spectra. Tellus 33, 411-414.

Leith, C. E. 1971. Atmospheric predictability and twodimensional turbulence. J. Atmos. Sci. 28, 145-161.

Leith, C. E. and Kraichnan, R. H. 1972. Predictability of turbulent flows. J. Atmos. Sci. 29, 1041-1058.

Lilly, D. K. 1983. Stratified turbulence and the mesoscale variability of the atmosphere. J. Atmos. Sci. 40, 749-761.

Lilly, D. K. 1989. Two-dimensional turbulence generated by energy sources at two scales. J. Atmos. Sci. 46, 2026-2030.

Lorenz, K. 1969. The predictability of a flow which possesses many scales of motion. Tellus 21, 289-307.

McWilliams, J. C. and Chow, J. H. S. 1984. The emergence of isolated coherent vortices in turbulent flow. J. Fluid Mech. 146, 21-43.

Maltrud, M. E. and Vallis, G. K. 1991. Energy spectra and coherent structures in two-dimensional turbulence and beta-plane turbulence. J. Fluid Mech. 228, 321-342.

Morita, O. and Uryu, M. 1989. Geostrophic turbulence in a rotating annulus of fluid. J. Atmos. Sci. 46, 2349-2355.

Nakamura, H. and Wallace, J. M. 1993. Synoptic

Tellus 51A (1999), 5 
behavior of baroclinic eddies during the blocking onset. Mon. Wea. Rev. 121, 1892-1903.

Nastrom, G. D. and Gage, K. S. 1985. A climatology of atmospheric wavenumber spectra observed by commercial aircraft. J. Atmos. Sci. 42, 950-960.

Orszag, S. A. 1977. Lectures on the statistical theory of turbulence. In: Fluid dynamics 1973, Les Houches Summer School of Theoretical physics (eds. Balian, R and Peuve, J.-L.). New York: Gordon and Breach, pp. 237-374.

Panetta, R. Lee. 1993. Zonal jets in wide baroclinically unstable regions: persistence and scale selection J. Atmos. Sci. 50, 2073-2105.

Rhines, P. B. 1975. Waves and turbulence on a $\beta$-plane. J. Fluid Mech. 69, 417-443.

Rhines, P. B. 1979. Geostrophic turbulence. Ann. Rev. Fluid Mech. 11, 401-441.

Salmon, R. 1980. Baroclinic instability and geostrophic turbulence. Geophys. Astrophys. Fluid Dyn. 15 167-212.

Saltzman, B. and Fleisher, A. 1960. The exchange of kinetic energy between larger scales of atmospheric motion. Tellus 12, 374-377.

Shepherd, T. G. 1987a. A spectral view of nonlinear fluxes and stationary-transient interactions in the atmosphere. J. Atmos. Sci. 44, 1166-1178.

Shepherd, T. G. 1987b. Rossby waves and two-dimen- sional turbulence in a large-scale zonal jet. J. Fluid Mech. 183, 467-509.

Steinberg, H. L. and Wiin-Nielsen, A. 1971. On nonlinear cascades in large-scale atmospheric flow. J. Geophys. Res. 76, 8629-8640.

Straus, D. M. 1983. On the role of the seasonal cycle J. Atmos. Sci. 40, 303-313.

Terry, P. W. and Newman, D. E. 1993. Nonconservative and reverse spectral transfer in Hasegawa-Mima turbulence. Phys. Fluids B 5, 2080-2085.

Trenberth, K. E. and Solomon, A. 1993. Implications of global atmospheric spatial spectra for processing and displaying data. J. Climate 6, 531-545.

Vallis, G. K. 1983. On the predictability of quasi-geostrophic flow: the effects of beta and baroclinicity. J. Atmos. Sci. 40, 20-27.

Vallis, G. K., Shutts, G. J. and Gray, M. E. B. 1997. Balanced mesoscale motion and stratified turbulence forced by convection. Quart. J. Roy. Meteor. Soc. 23, $1621-1652$.

White, G. H. 1982. An observational study of the Northern Hemisphere extratropical summertime general circulation. J. Atmos. Sci. 39, 24-40.

Wiin-Nielsen, A. 1967. On the annual variation and spectral distribution of atmospheric energy. Tellus, 19, 540-559.

Wiin-Nielsen, A. 1972. A study of power laws in the atmospheric kinetic energy spectrum using spherical harmonic functions. Meteor. Ann. 6, 107-124. 